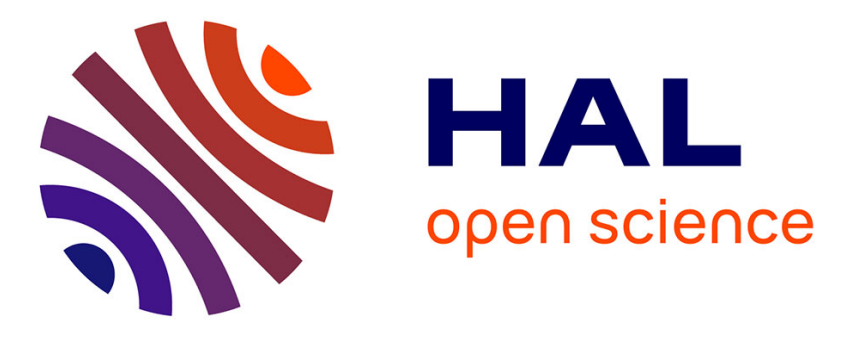

\title{
A Phelipanche ramosa KAI2 Protein Perceives enzymatically Strigolactones and Isothiocyanates
}

Alexandre de Saint Germain, Anse Jacobs, Guillaume Brun, Jean-Bernard Pouvreau, Lukas Braem, David Cornu, Guillaume Clavé, Emmanuelle Baudu, Vincent Steinmetz, Vincent Servajean, et al.

\section{To cite this version:}

Alexandre de Saint Germain, Anse Jacobs, Guillaume Brun, Jean-Bernard Pouvreau, Lukas Braem, et al.. A Phelipanche ramosa KAI2 Protein Perceives enzymatically Strigolactones and Isothiocyanates. 2020. hal-02997223

\section{HAL Id: hal-02997223 \\ https://hal.science/hal-02997223}

Preprint submitted on 10 Nov 2020

HAL is a multi-disciplinary open access archive for the deposit and dissemination of scientific research documents, whether they are published or not. The documents may come from teaching and research institutions in France or abroad, or from public or private research centers.
L'archive ouverte pluridisciplinaire HAL, est destinée au dépôt et à la diffusion de documents scientifiques de niveau recherche, publiés ou non, émanant des établissements d'enseignement et de recherche français ou étrangers, des laboratoires publics ou privés. 
1 A Phelipanche ramosa KAI2 Protein Perceives enzymatically Strigolactones and

2 Isothiocyanates

3 Alexandre de Saint Germain, ${ }^{a}$ Anse Jacobs, ${ }^{\text {b,c,dee }}$ Guillaume Brun, ${ }^{\text {f,g }}$ Jean-Bernard Pouvreau, ${ }^{f}$

4 Lukas Braem, ${ }^{\mathrm{b}, \mathrm{c}, \mathrm{d}, \mathrm{e}}$ David Cornu, ${ }^{\mathrm{h}}$ Guillaume Clavé, ${ }^{\mathrm{i}}$ Emmanuelle Baudu, ${ }^{\mathrm{a}}$ Vincent Steinmetz, ${ }^{\mathrm{i}}$

5 Vincent Servajean, ${ }^{\mathrm{i}}$ Susann Wicke, ${ }^{\mathrm{g}}$ Kris Gevaert, ${ }^{\mathrm{d}, \mathrm{e}}$ Philippe Simier, ${ }^{\mathrm{f}}$ Sofie Goormachtig, ${ }^{\mathrm{b}, \mathrm{c}}$

6 Philippe Delavault ${ }^{\mathrm{f}}$ and François-Didier Boyer ${ }^{\mathrm{i}^{*}}$

7 * Correspondence to: François-Didier Boyer, E-mail: francois-didier.boyer@cnrs.fr

8 a Institut Jean-Pierre Bourgin, Université Paris-Saclay, Institut National de Recherche pour

9 l'Agriculture, l'Alimentation et l'Environnement, AgroParisTech, 78000, Versailles, France.

$10{ }^{\mathrm{b}}$ Department of Plant Biotechnology and Bioinformatics, Ghent Univeristy, 9052 Gent, 11 Belgium.

$12{ }^{\mathrm{c}}$ Center for Plant Systems Biology, VIB, 9052 Gent, Belgium.

$13{ }^{\mathrm{d}}$ Department of Biomolecular Medicine, Ghent University, 9000, Ghent, Belgium.

$14{ }^{\mathrm{e}}$ Center for Medical Biotechnology, VIB, 9000 Ghent, Belgium.

$15{ }^{\mathrm{f}}$ Laboratoire de Biologie et Pathologie Végétales (LBPV), Equipe d'Accueil 1157, Université 16 de Nantes,44000, Nantes, France.

17 g Institute for Biology, Humboldt-Universität zu Berlin, 10115 Berlin, Germany.

$18{ }^{\mathrm{h}}$ Institute for Integrative Biology of the Cell (I2BC), Université Paris-Saclay, Centre d'Etudes

19 Atomiques, Centre National de la Recherche Scientifique, 91198, Gif-sur-Yvette, France.

$20{ }^{i}$ Institut de Chimie des Substances Naturelles, Unité Pédagogique Régionale 2301, Université 21 Paris-Saclay, Centre National de la Recherche Scientifique, 91198, Gif-sur-Yvette, France. 


\section{Abstract}

Phelipanche ramosa is an obligate root-parasitic weed threatening major crops in central Europe. For its germination, it has to perceive various structurally diverging host-exuded signals, including isothiocyanates (ITCs) and strigolactones (SLs). However, the receptors involved are still uncharacterized. Here, we identified five putative SL receptors in P. ramosa, of which PrKAI $2 \mathrm{~d} 3$ is involved in seed germination stimulation. We established the high plasticity of PrKAI2d3, allowing interaction with different chemicals, including ITCs. The SL perception mechanism of PrKAI2d3 is similar to that of endogenous SLs in non-parasitic plants. We provide evidence that the PrKAI2d3 enzymatic activity confers hypersensitivity to SLs. Additionally, we demonstrated that methylbutenolide-OH binds PrKAI2d3 and stimulates $P$. ramosa germination with a bioactivity comparable to that of ITCs. This study highlights that $P$. ramosa has extended its signal perception system during evolution, a fact to be considered in the development of specific and efficient biocontrol methods.

\section{Introduction}

Witchweeds (Striga spp.) and broomrapes (Orobanche and Phelipanche spp.) are obligate root-parasitic plants belonging to the Orobanchaceae family and together comprise the most threatening weeds of the major domesticated crops worldwide ${ }^{1}$. At maturity a single plant releases up to 100,000 microscopic seeds, resulting in severe soil pollution ${ }^{2}$. Seed germination of these obligate parasites requires the strict recognition of host-exuded germination stimulants. Broomrapes and witchweeds are all highly sensitive to strigolactones (SLs) secreted by plants into the rhizosphere at picomolar doses ${ }^{3}$. The structural core of SLs is a tricyclic lactone, referred to as the ABC part in canonical SLs or as a structural variety in noncanonical SLs, invariably connected to an $\alpha, \beta$-unsaturated furanone moiety (D ring) via an enol-ether bridge (Supplementary Figure $1 \mathbf{a})^{3}$. Some germination stimulants are exclusive to specific host-parasite interactions, as illustrated by the unique ability of Phelipanche ramosa to germinate upon sensing isothiocyanates (ITCs). These glucosinolate-breakdown products are exuded by rapeseed (Brassica napus) ${ }^{2,4}$, on which $P$. ramosa has adapted in a decade ${ }^{1,2}$. Broomrapes are increasingly problematic in both intensity and acreage in Europe, North Africa, and Asia, and they are expected to dramatically expand to new territories in the near future $^{5}$. To date, several physical, cultural, chemical, and biological approaches have been explored to control root-parasitic weeds, but no method has been found completely satisfactory ${ }^{6}$. 
Besides their involvement in germination, SLs have a strong stimulating activity on arbuscular mycorrhizal fungi (Glomeromycotina) by promoting mitochondrial metabolism and hyphal branching ${ }^{7}$, thereby mediating the establishment of the oldest mutualistic interaction of land plants $^{8}$. In addition to their role in rhizosphere signaling, SLs act as hormones in planta with pervasive roles throughout the plant development, ${ }^{9}$ and, as such, are perceived in vascular plants by the $\alpha / \beta$-hydrolase DWARF14 (D14) ${ }^{10,11}$. Biochemical analyses with recombinant D14 proteins by means of the synthetic SL analog GR24 revealed that the SL signal transduction requires GR24 cleavage ${ }^{11,12}$. One of the cleavage products, the $\mathrm{D}$ ring, may remain covalently attached to the receptor ${ }^{13,14}$, thereby probably allowing the recruitment of partners for downstream processes ${ }^{15,16}$. The required SL cleavage for signal transduction is still under debate ${ }^{15,16}$.

In Arabidopsis thaliana, D14 belongs to a small gene family, including KARRIKIN INSENSITIVE2/HYPOSENSITIVE TO LIGHT (AtKAI2/AtHTL) that shares the $\alpha / \beta$ hydrolase catalytic triad. However, AtKAI2 regulates AtD14-independent processes, such as seed germination, and preferentially perceives (-)-GR24 that mimics non-natural SLs, karrikins ${ }^{17}$, and supposedly a still unknown endogenous ligand ${ }^{18}$. Interestingly, the KAI2 gene family has expanded during the evolution of obligate parasitic-plant genomes ${ }^{19}$. For example, Phelipanche aegyptiaca and Striga hermonthica possess five and eleven KAI2/HTL genes, respectively. Two KAI2 paralogs play a role in SL response in $P$. aegyptiaca ${ }^{19}$ and six $S$. hermonthica KAI2/HTL proteins are hypersensitive to SLs ${ }^{19,20}$, with ShHTL7 exhibiting the same perception mechanism as $\mathrm{D} 14^{21}$. The remarkable expansion of the KAI2 gene family in Orobanchaceae along with the capacity of P. ramosa to perceive ITCs let us to assume that KAI2 proteins might also perceive other germination stimulants. Here, we characterize PrKAI2d3 as a P. ramosa SL receptor. We demonstrate that it is able to perceive natural SLs by an enzymatically dependent mechanism contributing to its hypersensitivity to SLs. In addition, we show that PrKAI2d3 perceives ITCs as well as a wide range of SL analogs that thus were not found bioactive for hormonal SL functions. Together, these results suggest that PrKAI2 proteins evolved as hypersensitive and plastic receptors, enabling the parasitic plant to detect various host exudate metabolites and contributing to its dramatic success.

\section{Results}

\section{Identification and gene expression profile of PrKAI2 homologs}


Iterative nucleotide and amino-acid BLAST analyses were carried out on the recently published transcriptome of $P$. ramosa rapeseed $\operatorname{strain}^{22}$, with known KAI2 and D14 sequences of non-parasitic and parasitic plants as queries ${ }^{19,20}$. Each newly identified sequence was used as a query for new BLAST searches to find all complete sequences. Duplicates were eliminated before alignment, allowing the detection of one $\operatorname{PrD} 14$ and five $\operatorname{PrKAI2}$ putative orthologs that were amplified and re-sequenced from cDNA isolated from germinated seeds of the same P. ramosa strain. Maximum-likelihood analysis indicated that the predicted PrD14 and PrKAI2 proteins (Figure 1a) and genes (Supplementary Figure 2) belonged to clearly distinct D14 and KAI2 clades, respectively, with a conserved catalytic triad and an overall conserved environment of known active-site amino-acid residues (Supplementary Figure 3). Despite a poor separation of non-parasitic and parasitic KAI2 groups, the phylogenetic analysis strongly suggested that among the retrieved transcripts no PrKAI2 protein belonged to the intermediate KAI2i subclade, as previously described for other members of the Orobanche and Phelipanche genera ${ }^{19}$. The newly identified PrKAI2 and PrD14 proteins consistently clustered together with $P$. aegyptiaca sequences. Together with the reported low levels of genomic divergence in Phelipanche $e^{23,24}$, these findings hint at the detection of $P$. aegyptiaca protein orthologs. Hence, the $P$. ramosa sequences were renamed according to the current nomenclature ${ }^{19}$, namely one PrKAI2 protein belonging to the conserved KAI2 (PrKAI2c) clade and four PrKAI2 proteins from the divergent KAI2 clade (PrKAI2d1-d4).

\section{PrKAI2d3 is a putative SL receptor}

Sequence homology analysis of the ligand-binding pocket amino-acid residues of the PrKAI2 proteins with AtD14 and AtKAI2 showed that PrKAI2d3 possesses the highest similarity with AtD14 (Figure 1b). Predictive models generated via the SWISS-MODEL webserver revealed that the binding pocket of PrKAI2c is smaller than that of its putative ortholog AtKAI2 (Figure 1c) and that the binding pockets of the PrKAI2d receptors are wider than those of members of the conserved KAI2 clade, probably due to the absence of large hydrophobic residues (Figure 1c). Among all the divergent receptors PrKAI2d3 had the largest predicted binding pocket (Figure 1c). Finally, the expression level of PrKAI2d3 transcripts in P. ramosa seeds was significantly up-regulated upon 1 hour of exogenous treatment with GR24, whereas the transcript levels for PrKAI2c, the putative AtKAI2 ortholog, did not change (Figure 1d). These properties are in agreement with a role of PrKAI2d3 as SL receptor. 
117 As no mutants and nor an easy and fast transformation method are available for holoparasitic 118 Orobanchaceae, it is difficult ${ }^{25}$ to validate the biological function of PrKAI2d 3 directly in $P$. 119 ramosa. Instead, we assayed the complementation of the Arabidopsis htl-3 mutant phenotype 120 with PrKAI2d3, a previously successful approach ${ }^{19,20}$. To assess the role of the catalytic triad 121 in the perception process, $h t l-3$ was also transformed with a mutated PrKAI $2 \mathrm{~d} 3{ }^{\mathrm{S} 98 \mathrm{~A}}$-encoding 122 version. We used three p35S::GFP-PrKAI2d3 lines displaying various protein levels and two p35S::GFP-PrKAI2d3 ${ }^{\text {S98A }}$ lines with a high protein expression (Figure 1e) to phenotype both germination and hypocotyl length.

In a thermo-inhibition assay ${ }^{26}$, almost no germination was detected for all lines upon control treatments. Germination of Arabidopsis (accession Columbia-0 [Col-0]) seeds was significantly stimulated upon $10 \mu \mathrm{M}(-)$-GR24, but not upon (+)-GR24, whereas none of the enantiomers had an effect on $h t l-3$ or Atd14-1/htl-3 mutants (Figure 1f). Germination of all three p35S::GFP-PrKAI2d3 lines was strongly induced by (+)-GR24. Exogenous (-)-GR24 also significantly stimulated germination of these three lines (Figure 1f) and the response amplitude correlated positively with the protein abundance (Figure 1e). Analysis of a fourth p35S::GFP-PrKAI2d3 line showed that even a mild protein expression increased the Arabidopsis sensitivity a 100-fold towards (-)-GR24, enabling it to perceive up to picomolar doses of (+)-GR24 and, hence, to have the P. ramosa sensitivity (Figure 2a-b, Supplementary Figure 4, Supplementary Table 1). None of the two p35S::GFP-PrKAI2d3 ${ }^{\text {S98A }}$ lines were sensitive to either $(+)-$ or $(-)-G R 24$.

137 Under red-light conditions, hypocotyls of $h t l-3$ and Atd14-1/htl-3 mutants are more elongated than those of Col- $0^{17}$ (Figure 1g). Application of $( \pm$ )-GR24 significantly shortened the hypocotyls of Col-0 and the htl-3 genotypes, but not those of the Atd14-1/htl-3 plants. This observation corroborates a previous report that seedling photomorphogenesis is redundantly controlled by D14 and KAI2/HTL in Arabidopsis ${ }^{17}$. Interestingly, only the hypocotyls of the p35S::GFP-PrKAI2d3 and p35S::GFP-PrKAI2d3 ${ }^{\text {S98A }}$ lines with the highest protein levels

143 were shorter than those of the $h t l-3$ and Atd14-1/htl-3 mutants under control conditions.

144 However, the hypocotyls were significantly shorterned in all transformed lines after treatment with $1 \mathrm{M}( \pm)$-GR24 (Figure 1g). In summary, these data indicate that the PrKAI2d3 protein expression rescues the $h t l-3$ mutant phenotypes, with the catalytic Ser98 being essential for germination, but not for seedling photomorphogenesis. 

analogs and mimics with various structures

As P. ramosa perceives many high structurally divergent germination stimulants, various receptors might underlie this plasticity ${ }^{27,28}$. To link the SL structural features with the $P$. ramosa seed germination activity, we carried out a structure-activity relationship (SAR) study through a rapid bioassay ${ }^{29}$.

154 First, we determined the P. ramosa sensitivity towards several GR24 analogs with varying stereogenic centers (Figure 2a). The lowest $\mathrm{EC}_{50}$ values were obtained with $(+)$-GR24 (6.5 $\mathrm{pM})$ and (-)-2'-epi-GR24 (5.3 pM), of which the stereochemistry corresponds to natural canonical SLs of the strigol-type and orobanchol-type series, respectively (Supplementary Figure 1a). In contrast, P. ramosa was approximately 100-fold less sensitive to (-)-GR24 and (+)-2'-epi-GR24, of which the stereochemistry is not encountered in natural SLs (Figure 2b, Supplementary Figure 1a).

161 Second, we investigated whether substitutions occurring on the D-ring alter P. ramosa 162 responses $^{27}$. Surprisingly, both GR24 analogs without a methyl group on the D-ring, $( \pm)-4$ '163 desmethyl-2'-epi-GR24 and ( \pm )-4'-desmethyl-GR24, significantly stimulated P. ramosa seed germination $\left(\mathrm{EC}_{50}=0.92\right.$ and $\left.0.45 \mathrm{nM}\right)$, still with 100 -fold lower $\mathrm{EC}_{50}$ values than those of $( \pm)$-GR24 (Figure 2c). Noteworthy, none of these substituted GR24 analogs could inhibit shoot branching even at $1 \mu \mathrm{M}^{27}$. Inversely, P. ramosa was less sensitive to ( \pm )-3'-methylGR24 $\left(\mathrm{EC}_{50}=2.6 \mathrm{nM}\right)$, which harbors two methyl groups on the D-ring (Figure 2c,

168 Supplementary Figure 5, Supplementary Table 2) and is highly bioactive in repressing pea 169 (Pisum sativum) shoot branching via RMS3/PsD14 ${ }^{13,27}$.

170 Finally, the effect of the ABC-ring fragment substitution on the P. ramosa germination was analyzed. Profluorescent probes were used, in which the ABC-ring was replaced by a coumarine moiety (DiFMU) and one, two, or no methyl groups were added on the D-ring

173 (GC240, GC242, and GC486, respectively ${ }^{13}$. In another profluorescent probe, the ABC-ring 174 was switched by the fluorescein moiety Yoshimulactone green (YLG) ${ }^{30}$ (Supplementary 175 Figure 1b). Except for DiFMU, all profluorescent probes had a stimulating activity on the $P$. 176 ramosa germination, but still with 1,000- to 10,000-fold lower $\mathrm{EC}_{50}$ values than those of $( \pm)$ 177 GR24 (Supplementary Figure 6a-c, Supplementary Table 2). 
178 Altogether, these results demonstrate that the stereochemistry of GR24 analogs is crucial for 179 bioactivity and determines the sensitivity to germination-inducing substances. The sensitivity 180 of $P$. ramosa towards desmethyl and 3'-methyl D-ring derivatives highlights differences in contrast to other vascular plants, particularly regarding the signaling via D14 for shoot

182 branching. Additionally, the relative bioactivity of the profluorescent SL probes for 183 germination stimulation in broomrape confirms that $\mathrm{ABC}$-rings are not required for 184 bioactivity $^{28}$.

SL analogs and mimics interact with PrKAI2d3 according to their germination

\section{stimulation activity}

187 The questions arising from previous results are whether PrKAI2d3 has the ability to perceive such a large range of structurally divergent compounds and whether its interaction with these molecules is affected by mutations in the catalytic triad. To this end, we expressed and purified the $\operatorname{PrKAI} 2 \mathrm{~d} 3$ and $\operatorname{PrKAI} 2 \mathrm{~d}^{\mathrm{S}}{ }^{\mathrm{S}}{ }^{\mathrm{A}}$ proteins in vitro and assessed their abilities to interact with SLs and other chemical mediators.

Interactions between PrKAI2d3 and the SL analogs and profluorescent SL probes were analyzed with nano differential scanning fluorimetry (nanoDSF) by recording changes in the

194 tryptophan fluorescence (ratio $350 \mathrm{~nm} / 330 \mathrm{~nm}$ ). In contrast to "classical" DSF", nanoDSF 195 does not require a dye and highlights interactions that do not induce conformational changes.

196 Analysis of the initial fluorescence ratios revealed that the four GR24 stereoisomers interacted with PrKAI2d3 according to their bioactivity (Figure 2d). However, only (+)-GR24 and (-)2'-epi-GR24, the most bioactive analogs with natural configurations, induced a $8.5{ }^{\circ} \mathrm{C}$

199 decrease in the PrKAI2d3 melting temperature (Figure 2e), consistent with ligand-mediated 200 protein destabilization.

201 When the GR24 analogs with varying methyl groups on their D-ring were used, all the analogs interacted and destabilized PrKAI2d3, although with an efficiency lower than that of $( \pm)$-GR24, and especially that of $( \pm$ )-3'-Me-GR24 (Figure 2f,g). Similar shifts in melting

204 temperatures of the PrKAI2d3 protein were observed with DSF, but without destabilization of 205 the mutated PrKAI2d3 ${ }^{\text {S98A }}$ protein (Supplementary Figure 7). The bioactive profluorescent 206 probe GC242, used as a racemic mixture, or separate pure enantiomers also induced a shift in 207 the PrKAI2d3 protein melting temperature (Supplementary Figure 6d-i). These results 208 suggest that the enzymatic activity through Ser98 is required to destabilize the protein. 
Next, we estimated the PrKAI2d3 affinity for GR24 analogs by means of the tryptophan intrinsic fluorescence assay. The affinity was higher for (+)-GR24 and (-)-2'-epi-GR24 ( $K_{\mathrm{D}}=$

$21112.57 \pm 2.85 \mu \mathrm{M}$ and $66.99 \pm 26.94 \mu \mathrm{M}$, respectively) than for (-)-GR24 and (+)-2'-epi212 GR24 $\left(K_{\mathrm{D}}=696 \pm 245 \mu \mathrm{M}\right.$ and $434 \pm 593 \mu \mathrm{M}$, respectively), in accordance with their 213 bioactivity on the P. ramosa germination (Figure $2 \mathbf{h}-\mathbf{k}$, Supplementary Figure 8), whereas the 214 lower affinity of $( \pm)-3$ '-Me-GR24 than that of ( \pm )-4'-desmethyl-2'-epi-GR24 and ( \pm -GR24 is consistent with their reduced bioactivity range (Figure 2l). However, protein affinities were

216 significantly lower (micromolar range) than the observed sensitivity of $P$. ramosa (picomolar 217 range). As similar patterns had been reported for Striga hermonthica ${ }^{30}$, we tested this apparent 218 contradiction by investigating the enzymatic activity of the PrKAI2d3 protein.

The PrKAI2d3 enzymatic activity is associated with SL hypersensitivity

First, we tried to visualize the PrKAI2d3 hydrolase activity with a generic substrate, paranitrophenyl acetate ( $p$-NPA). Surprisingly, no hydrolytic activity was detected (Supplementary Figure 10) in contrast to other SL receptors with the same probe ${ }^{13}$. When PrKAI2d3 was incubated with (+)-GR24 and (-)-2'-epi-GR24, a cleavage activity was unambiguously observed by ultraperformance liquid chromatography (UHPLC)/UV DAD analysis (Figure 3a), but, opposite to AtD14 and AtKAI2, PrKAI2d3 did not cleave (-)-GR24 or $(+)-2$ '-epi-GR24. Interestingly, a residual cleavage activity of PrKAI2d3 ${ }^{\text {S98A }}$ still occurred with all GR24 isomers, but without stereoselectivity, possibly the reason for the partial complementation of $h t l-3$ with PrKAI2d $3^{\mathrm{S} 98 \mathrm{~A}}$-overproducing constructs (Figure 1g). These data suggest that SL hydrolysis and the subsequent signal transduction could happen without an intact catalytic triad.

As for the interaction assays, we tested the hydrolysis of analogs with substitutions on the Dring. Desmethyl GR24 isomers were more efficiently hydrolyzed than GR24 by AtKAI2, PrKAI2d3, and PrKAId $3{ }^{\text {S98A }}$ proteins (Figure 3b). Additionally, these three purified proteins along with AtD14 also displayed a low, but significant, hydrolysis activity towards 3'-MeGR24. These results indicate that PrKAI2d3 possesses an important hydrolysis capacity towards the natural configuration-mimicking GR24, albeit to a lesser extent than AtD14. bioactive profluorescent probes as substrate. Monitoring the DiFMU fluorescence revealed that PrKAI2d3 hydrolyzed $( \pm)-G C 240,( \pm)-G C 242$, and $( \pm)$-GC486 (Figure 3c-e). For all 
probes, we observed a biphasic time course of fluorescence, consisting of a burst phase (also called initial phase), followed by a plateau phase (or slow phase for AtD14 incubated with

$242( \pm)-G C 240)$. In all cases, the plateau did not reach the maximum of the expected product $243(20 \mathrm{M})$, but a concentration product closer to the protein concentration (0.4 M) (Figure 3c-e), 244 implying that PrKAI2d3 might act as a single turnover enzyme towards all GC probes tested, 245 including GC486 that lacks the 3'-methyl group on the D-ring and has a Michaelian cleavage 246 kinetic with AtD14 and RMS3/PsD14 proteins $^{13}$. The S98A substitution in the catalytic triad 247 drastically reduced the $( \pm)$-GC240 and $( \pm)$-GC242 cleavage, although the residual activity 248 towards $( \pm)$-GC486 remained statistically significant. These observations confirmed that the 249 mutation in the catalytic triad does not fully abolish the cleavage activity towards a compound 250 without methyl on the D-ring.

251 Regarding single turnover enzymes, we defined $k_{\text {cat }}$ as the rate constant of the presteady-state 252 phase (initial phase) and $K_{1 / 2}$ as the probe concentration with half maximal velocity $\left(V_{\max }\right)$ 253 (Figure 3g-i). The similar $K_{1 / 2}$ values of PrKAI2d3 with ( \pm )-GC240 and ( \pm )-GC242 $(5.74 \mu \mathrm{M}$ 254 and $4.60 \mu \mathrm{M}$, respectively) confirmed the low influence of the C3' methyl chain on the 255 substrate affinity, but the differences in the $V_{\max }\left(0.072 \mathrm{M} \cdot \mathrm{min}^{-1}\right.$ and $0.013 \mathrm{M} \cdot \mathrm{min}^{-1}$, 256 respectively) indicated that the $\mathrm{C} 3$ ' methyl chain reduces the catalytic activity, corresponding 257 with the bioactivities of these probes. Higher $K_{1 / 2}$ values of PrKAI2d3 for $( \pm)$-GC240 than 258 that for $( \pm)$-GC486 $\left(5.74 \mu \mathrm{M}\right.$ and $1.53 \mu \mathrm{M}$, respectively) and $V_{\max }$ values $\left(0.072 \mathrm{M} \cdot \mathrm{min}^{-1}\right.$ and $2590.209 \mathrm{M} \cdot \mathrm{min}^{-1}$, respectively) highlighted the importance of the C4' methyl chain in binding 260 and catalytic affinity, supporting the results of the germination bioassays (Supplementary 261 Figure 6a-c).

262 To test the hypothesis that PrKAI2d3 forms a stable intermediate with the D-ring, as 263 previously demonstrated for other SL receptors (AtD14, PsD14/RMS3, ShHTL7, and 264 D14) $)^{13,14,21,31}$, we incubated two bioactive ligands ( \pm )-GR24 and probe $( \pm)$-GC486 with 265 PrKAI $2 \mathrm{~d} 3$ at a $\mathrm{pH}$ of 6.8 and recorded mass spectrometry spectra under denaturing 266 conditions. In all cases a mass shift occurred corresponding to the D-ring covalently bound to 267 the protein (Figure $3 \mathbf{j}-\mathbf{l}$ ) and specifically attached to His 249 of the catalytic triad (Figure $3 \mathbf{m}$ 268 o). 
Although PrKAI2d3 perceives structurally diverging SL analogs and mimics that stimulate seed germination of $P$. ramosa, the specificity of the interaction between rapeseed and $P$. ramosa correlates with the parasite's ability to perceive ITCs, which structurally differ greatly from SLs ${ }^{4}$. Therefore, we evaluated the ability of PrKAI2d 3 to perceive ITCs. Seeds of $P$. ramosa were approximately 10,000-fold less sensitive to 2-PEITC and BITC than to $( \pm)$ GR24 (Figure 4a-c). Investigation of the putative interactions between PrKAI2d3 and ITCs by nanoDSF revealed a small shift $\left(1.2-2.0^{\circ} \mathrm{C}\right)$ in the PrKAI $2 \mathrm{~d} 3$ melting temperature upon high ITC concentrations, as further confirmed by "classical DSF" (Figure 4d-e, Supplementary Figure 11). These data indicated that ITCs interact with PrKAI2d3 with low affinity, corresponding with a stimulating potential on P. ramosa seeds lower than that of bioactive $\mathrm{SLs}^{4}$ and analogs. Finally, the apparent melting temperatures of PrKAI2d 3 with BITC and 2-PEITC $\left(44.3{ }^{\circ} \mathrm{C}\right.$ and $45.1^{\circ} \mathrm{C}$, respectively) varied from values obtained with $( \pm)$ GR24 $\left(36.9^{\circ} \mathrm{C}\right)$, suggesting that ITCs might induce a conformational change that differs from the GR24-induced destabilization.

As ITCs easily react with nucleophile functions ${ }^{32}$, we hypothesized that the PrKAI2d3 interaction with ITCs may trigger the formation of a covalent adduct. Indeed, a mass shift was detected correlating to the 2-PEITC covalently bound to the protein (Figure $4 \mathbf{f}$ ). After the PrKAI2d3-2-PEITC complex digestion, the 2-PEITC attachment was localized on a peptide corresponding to the amino acids $87-113$ of PrKAI2d3. Tandem mass spectrometry data revealed that the 2-PEITC attachment could be on His97 or on the catalytic Ser98 (Figure 4h). Incubation of 2-PEITC with PrKAI2d3 ${ }^{\text {S98A }}$ allowed us to conclude that the major site for 2PEITC attachment is on Ser98 (Figure 4g). These results hint at a perception mechanism for ITC in which the Ser98 hydroxyl group would react with isothiocyanate to generate a PrKAI2d3-attached carbamothioate (Figure 4i). However, no 2-PEITC attachment was detected on His249-containing peptides of the catalytic triad.

Overall, PrKAI2d3 acts as a receptor for both SL-like molecules and ITCs that are both germination stimulants of $P$. ramosa. These results demonstrate that further research into potential chemical interactors is achievable to design control methods.

Search for small-molecule interactors with PrKAI2d3 proposes D-OH as a simple and efficient germination stimulant for $P$. ramosa with a bioactivity similar to that of ITCs 
300 Recently, synthetic inhibitors of D14 and ShHTL $7^{33}$, the $S$. hermonthica SL receptor, have been proposed, that include, although all structurally unrelated to SLs, soporidine, KK094, Triton X, and tolfenamic acid (TA) (Figure 5a). We evaluated the putative inhibitory effects 303 of these compounds, along with those of a common serine protease inhibitor, 304 phenylmethylsulfonyl fluoride (PMSF) on the germination of P. ramosa seeds treated with $10 \mathrm{nM}$ of $( \pm)-G R 24$ or $100 \mathrm{nM}$ of 2-PEITC. Nonetheless, P. ramosa seeds were clearly hyposensitive to each of these compounds when compared to results obtained in $S$. hermonthica, with a maximum inhibition obtained in some instances at very high concentrations (Figure 5b,c; Supplementary Table 3). Indeed, all half maximum inhibitory concentrations $\left(\mathrm{IC}_{50}\right)$ fell above the $\mathrm{IC}_{50}$ of abscisic acid (ABA, $100 \mathrm{nM}$ (GR24) and $34 \mathrm{nM}$ (2-PEITC)], a known inhibitor of P. ramosa germination ${ }^{34,35}$. These data indicate that the use of germination inhibitors intended for Striga is reasonably irrelevant and unsuitable for $P$. ramosa biological control.

313 Therefore, we looked for specific PrKAI2d3 agonists with the potential for large-scale 314 biocontrol. A serious issue in the search for simple and cheap SL analogs and mimics is their 315 low stability due to the D-ring connection to a leaving group. As alternatives, molecules have 316 been proposed that carry the D-ring only, because it is essential for the high bioactivity of $317 \mathrm{SLs}^{36}$. Specifically, the effect on the P. ramosa germination was evaluated of a set of 318 butenolides containing the D-ring (D-OR) only (Figure 5j, Supplementary Figure 12). D$319 \mathrm{O} \sec \mathrm{Bu}$ and 3'-Me-D-OH were completely inactive, whereas the P. ramosa sensitivity towards D-OAll, D-OMe, D-OEt, and Dihydro-DOEt was low with $\mathrm{EC}_{50}$ in the micromolar range. Inversely, D-OH and 4'-Desmethyl-D-OH were approximately 10-fold more active than 2-PEITC (Supplementary Table 2). Although D-OH had been found bioactive in rice (Oryza sativa) at high concentration ${ }^{12}(50 \mu \mathrm{M})$, in pea D-OH and 3'-Me-D-OH had not effect on the branching control by direct injection $(100 \mu \mathrm{M})$ in the stem (Supplementary Figure 13).

To check whether this D-OH sensitivity was a specificity of the P. ramosa PrKAI2d3, we reexamined the germination-stimulating activity of $\mathrm{D}-\mathrm{OH}$ on $S$. hermonthica. In contrast to previous results ${ }^{37,38}, \mathrm{D}-\mathrm{OH}$ induced $S$. hermonthica germination $\left(\mathrm{EC}_{50}=1.2 \mu \mathrm{M}\right)$. Moreover, in Arabidopsis lines expressing a GFP fused to ShHTL7, the known S. hermonthica SL receptor $^{30,39}$, was induced by $( \pm)-\mathrm{DOH}\left(\mathrm{EC}_{50}=3.1 \mu \mathrm{M}\right)$ (Supplementary Figure 14,

330 Supplementary Table 1). In summary, our study validates the perception of D-OH by SL receptors in root-parasitic plants. 
DSF and intrinsic fluorescence analyses revealed that D-OH and PrKAI2d 3 interacted at high concentrations (Figure 5k-l). Incubation of D-OH with PrKAI2d3 at pH 6.8 and the recorded MS spectra under denaturing conditions revealed a mass shift corresponding to the D-ring covalently bound to the protein (Figure $5 \mathbf{m}$ ). The D-ring attachment could be localized to the His249 of the catalytic triad, similarly as for the SL analogs (Figure 5n). In addition to various SL analogs, and SL-like compounds, and ITCs, D-OH highlights the plasticity of the PrKAI $2 \mathrm{~d} 3$ receptor to interact with different structures with a significant biological activity as a consequence and emphasizes that all SL hydrolysis products are not inactive as germination stimulants of $P$. ramosa.

\section{Discussion}

342 Obligate parasitic weeds require host-derived signals to germinate and wither their hosts long

343 before they emerge from the soil, arguably making the early stages of the parasitic life cycle a 344 much better target for control strategies than the later ones ${ }^{40}$. An important prerequisite for the 345 design of such methods is an in-depth understanding of how parasites perceive germination stimulants.

347 Here, we demonstrated that PrKAI2d3 provides $P$. ramosa with hypersensitivity to SLs mainly due to its enzymatic activity. The enzymatic data with the GR24 analogs [(+)-GR24 and (-)-2'-epi-GR24] and GC probes suggest that PrKAI2d3 acts as a single turnover enzyme towards natural SLs, and cleave them to form a covalent complex between the D-ring and the catalytic histidine, as previously described for AtD14 and RMS3 ${ }^{13}$. However, this model has recently been challenged ${ }^{16}$, because the Arabidopsis SL receptor mutated for the catalytic Asp residue, speculatively considered unable to cleave endogenous SLs, had been found to

354 transduce the SL signal; hence, the SL cleavage has been concluded not be required for signaling. Indeed, It is possible that certain SL analogs, can be perceived independently of the enzymatic activity. We showed that the GR24 analogs with non-natural stereochemistry [(-)GR24 and (+)-2'-epi-GR24] are not cleaved by PrKAI2d3. These uncleaved molecules stimulate 100-fold less efficiently the $P$. ramosa seed germination than the GR24 stereoisomers with a natural stereochemistry (Figure 3). Moreover, the PrKAI2d3 affinity $360\left(K_{\mathrm{D}}\right)$ towards the most bioactive SL analogs, (+)-GR24 and (-)-2'-epi-GR24, is in the 361 micromolar range, which is several orders lower than the P. ramosa seed sensitivity recorded 362 in the germination bioassays. The $K_{\mathrm{D}}$ values for (+)-GR24 and (-)-2'-epi-GR24, that reflect a 
simple binding to the receptor, do not allow explaining the hypersensitivity. Similar differences between in vitro and in vivo analyses were observed for the $S$. hermonthica SL receptor ShHTL7 characterization ${ }^{30}$. We propose that the SL hypersensitivity of this SL receptor is obtained by its enzymatic properties.

367 Interestingly, because the enzymatic activity is not completely abolished in the PrKAI2d3 ${ }^{\text {S98A }}$ 368 protein, a residually significant activity, also present in the DAD2 ${ }^{\mathrm{S} 96 \mathrm{~A}}$ protein $^{41}$, might result 369 from the nucleophilic addition of water instead of the attack of the Ser98 hydroxyl group. The partial complementation of the $h t l-3$ mutant with constructs that overproduce PrKAI $2 \mathrm{~d} 3^{\mathrm{S} 98 \mathrm{~A}}$ corroborates this assumption. A similar proposition could also explain why Arabidopsis overexpressing the AtD14 with a mutated Asp catalytic residue is able to transduce a branching inhibitor signal, especially the still untested natural SLs present in Arabidopsis (i.e. non-canonical SLs). Noteworthy, $p$-NPA could not efficiently highlight the enzymatic activity of PrKAI2d3, in comparison to the profluorescent SL mimics, pinpointing the weakness of using generic or inappropriate substrates as controls.

Moreover, PrKAI2d3 displays a high plasticity that allows binding of modified SLs, such as desmethyl-GR24 isomers or many SL mimics, but in contrast to RMS3 and AtD14, it perceives these compounds similarly as intact SLs. Correspondingly, desmethyl-YLG was bioactive in Arabidopsis via AtKAI2 ${ }^{42}$. Ligand-mediated protein destabilization is not required to perceive SL analogs, but considerably improves the sensitivity to natural SLs.

382 PrKAI2d3 acts as single-turnover enzyme for compounds with a D-ring without methyl group at the C4' position, but a methyl group at the C-3' position led to low interaction with PrKAI2d3. This result is in accordance with the low germination stimulant activity of 3'methyl analogs and mimics for P. ramosa and corroborates studies conducted in O. cumana, O. minor, P. aegyptiaca, and S. hermonthica ${ }^{28,43}$. In other words, P. ramosa might be sensitive to D-ring modified SL and could perceive diverse SL-related compounds as putative SL degradation products ${ }^{44}$.

In addition, we established that ITCs, other known germination stimulants, interact with the PrKAI $2 \mathrm{~d} 3$ protein by forming a covalent complex. In P. ramosa, PrKAI $2 \mathrm{~d} 3$ can be considered as a germination stimulant receptor for different chemicals, including SLs, SL derivatives,

392 and mimics, but also ITCs with completely different structures. Thus, P. ramosa has 393 seemingly optimized its germination capacity by its ability to perceive various chemical 394 mediators emitted by the host plants. The enzymatic activity-dependent ITC perception 
mechanism suggests that the active catalytic triad might have been conserved, not only to perceive SLs, but also other germination stimulants, not yet characterized for P. ramosa.

397 SLs are very unstable in the soil, especially under basic conditions that lead to $\mathrm{ABC}=\mathrm{CHOH}$ 398 and D-OH derivatives. To date, all SL analogs and mimics possess a D-ring connected to an 399 ABC mimic by a hydrolysable function. The treatment with SL agonists developed until now 400 induces a suicide germination of plant-parasitic seeds ${ }^{45}$. Nevertheless, these agonists are too 401 unstable under field conditions to be effective, even when promising chemicals have been 402 designed and applied for S. hermonthica ${ }^{46}$. In addition, cultures of P. ramosa-infested 403 rapeseed mostly occur in slightly basic soils ${ }^{47}$, both favoring the formation of ITCs ${ }^{4}$ and 404 complicating the use of SL mimics as a suicidal germination strategy, especially due to their 405 instability. In sharp contrast, P. ramosa is highly sensitive to D-OH or DesMe-D-OH. As for 406 D-H, no germination activity was detected, the hemiacetal function seems very important for 407 the interaction with PrKAI2d3. Moreover, the residual bioactivity of D-OR $(\mathrm{R} \neq \mathrm{H})$ might be 408 explained by the hydrolysis of the R group during the bioassay, leading to D-OH. D-OH or 409 DesMe-D-OH are especially interesting for translational research, because both compounds 410 are small, are easily synthesized in comparison with synthetic SLs and SL mimics, and are not 411 subjected to fast degradation. As such, D-OH and DesMe-D-OH seem to be promising 412 chemicals for suicide germination in the field: their lower germination activity than that of SL 413 mimics can be compensated by higher active concentration. Moreover, D-OH has the great 414 advantage of being a natural product. The use of SL mimics, for which the part equivalent to 415 the $\mathrm{ABC}$ moiety is in most cases xenobiotic, increases the risk of potential toxicity or 416 pollution for the environment. In contrast to previous studies ${ }^{37,38}$, we found that $\mathrm{D}-\mathrm{OH}$ is 417 bioactive as a witchweed seed germination stimulant and that it could possibly be used for $S$. 418 hermonthica biocontrol.

419 Here, we identified germination inhibitors and demonstrated the involvement of $\alpha / \beta$ 420 hydrolases for germination-stimulating reception, providing an alternative track to fight $P$. 421 ramosa $^{48}$. Novel more active inhibitors need to be designed for P. ramosa infection control 422 without negative effects on the host plants and arbuscular mycorrhizal fungi. For this purpose, 423 the identification of the SL receptor(s) in these fungi will undoubtedly be an important 424 milestone. The use of specific inhibitors should be combined with other approaches for 425 integrated management strategies ${ }^{49}$, such as chemical suicidal germination with compounds, 426 such as D-OH, to decrease the parasitic-plant seed bank in soil. 
Preparation of GR24 isomers, probes, and other ligands. For general experimental procedures, see the Supplementary Methods. $( \pm)-2$ '-epi-GR24 and ( \pm )-GR24 were prepared as 430 described $^{50}$ and $( \pm)$-4-desmethyl-GR24, $( \pm)$-2'-epi-4-desmethyl-GR24, and $( \pm)$-3'-Me-GR24 431 as described ${ }^{27}$. (+)-GR24, (-)-GR24, (+)-2'-epi-GR24, and (-)-2'-epi-GR24 were separated 432 from ( \pm )-2'-epi-GR24 and ( \pm )-GR24 by chiral supercritical fluid chromatography as 433 described ${ }^{13,51} .( \pm)$-GR24 was purified by semi-preparative HPLC by means of a Interchim 434 puriFlash ${ }^{\circledR} 4250$ instrument, combined with a fraction collector with integrated ELSD, a 435 PDA and a Phenomenex Luna C18, $250 \times 21.2 \mathrm{~mm}, 5-\mu \mathrm{m}$ column $\left(\mathrm{H}_{2} \mathrm{O} / \mathrm{CH}_{3} \mathrm{CN}\right.$ : 6/4) or 436 Interchim Uptisphere Strategy SI, $250 \times 21.2 \mathrm{~mm}, 5-\mu \mathrm{m}$ column (Heptane/EtOAc: 1/1). 2 437 Phenethyl isothiocyanate (2-PEITC), benzylisothiocyanate (BEITC), DiFMU, para438 nitrophenyl acetate ( $p$-NPA), Yoshimulactone Green (YLG), abscisic acid (ABA), tolfenamic $439 \operatorname{acid}^{52}$ (TA), TritonX-100 53 and phenylmethylsulfonyl fluoride (PMSF) are commercially 440 available. KK094 $4^{54}$ and soporidine ${ }^{55}$ were kindly provided by T. Asami and P. McCourt 441 (University of Toronto), respectively. Probes (GC486, GC240, and GC242) were prepared as 442 described $^{13}$. For the preparations of D-OH and analogs, see Supplementary Methods.

443 Expression and purifications. AtD14 and AtKAI2 were purified and expressed with 444 cleavable GST tags as described ${ }^{13}$. For PrKAI2d3 expression, the coding sequences from 445 Phelipanche ramosa were amplified by PCR by means of a seed-derived cDNA template and 446 specific primers (Supplementary Table 4) containing a protease cleavage site for tag removal, 447 and subsequently cloned into the pGEXT-4T-3 expression vector. The PrKAI2d3 and $448 \quad \operatorname{PrKAI} 2 \mathrm{~d}^{\mathrm{S} 98 \mathrm{~A}}$ proteins were purified and expressed as above.

449 Site-directed mutagenesis. Site-directed mutagenesis experiments were done with the 450 QuickChange II XL Site Directed Mutagenesis kit (Stratagene) on pGEX-4T-3-PrKAI2d3 451 (Supplementary Table 4). Mutagenesis was verified by systematic DNA sequencing.

452 Plant material and growth conditions. Pea (Pisum sativum) branching mutant plants were 453 derived from various cultivars after ethyl methanesulfonate (EMS) mutagenesis and had been 454 described previously ${ }^{56}$. The $r m s 1-10$ (M3T-884) mutant was obtained from the dwarf cv 455 Térèse. Plants were grown in a greenhouse under long-day as described ${ }^{57}$. 
All Arabidopsis thaliana (L.) Heynh. mutant plants (Columbia-0 [Col-0] accession background) have been described previously: htl-3 (ref. ${ }^{58,59}$ ), Atd14-1/htl-3 (ref. ${ }^{58,59}$ ), and htl-3 ShHTL7 (kind gift of P. McCourt). For overexpression of the green fluorescent protein (GFP) fusions of PrKAI2d3 and PrKAI2d3 ${ }^{\text {S98A }}$, transgenic Arabidopsis seeds were generated 460 by the Agrobacterium floral dip method ${ }^{60}$ with the $h t l-3$ mutant as the background accession. Transgenic seeds were selected based on the antibiotic resistance and GFP fluorescence.

462

463

464

465

466

467

468

469

470

471

472

473

474

475

476

477

Two batches of parasitic plant seeds were used. A population of seeds of Phelipanche ramosa (L.) Pomel associated to the genetic group 1 (P. ramosa 1) was collected from Saint Martinde-Fraigneau (France) on broomrape-parasitizing winter rapeseed (Brassica napus L.) in 2014 and 2015 (ref. ${ }^{61}$ ). Seeds of Striga hermonthica (Delile) Benth. (Sudan, 2007) were provided by Lukas Spichal (Olomuc, Czech Republic). Seeds were surface sterilized and conditioned as described ${ }^{29}$ (darkness; $21^{\circ} \mathrm{C}$ and $30^{\circ} \mathrm{C}$ for P. ramosa and $S$. hermonthica, respectively).

Pea shoot-branching assay. The compounds to be tested were applied by vascular supply. The control was the treatment with $0.1 \%$ dimethylsulfoxide (DMSO) only. Twelve plants were sown per treatment in trays and generally 10 days after sowing the axillary bud at node 3 was treated. Compounds in DMSO solution were diluted in water to the indicated concentrations for a treatment with $0.1 \%(\mathrm{v} / \mathrm{v})$ DMSO. The branches at nodes 1 and 2 were removed to encourage the outgrowth of axillary buds at the nodes above. Nodes were numbered acropetally from the first scale leaf as node 1 and cotyledonary node as node 0 . Bud growth at nodes 3 and 4 was measured with digital callipers 8 to 10 days after treatment. Plants with damaged main shoot apex or with a dead white treated-bud were discarded from the analysis. The SL-deficient $r m s 1-10$ pea mutant was used for all experiments.

Cloning and generation of transgenic lines. For all GFP fusion constructs, cloning was done by Gateway recombination (Thermo Fisher Scientific). The open reading frame (ORF) of PrKAI2d3 was amplified from P. ramosa cDNA with iProof ${ }^{\mathrm{TM}}$ High-Fidelity DNA Polymerase (Bio-Rad) and the Gateway®-specific primers PrKAI2d3_B2_FW and PrKAI2d3_B3_Rev_STOP. The PCR product flanked by the attB sites was cloned in pDONR P2R-P3 with the BP Clonase II enzyme mix (Invitrogen). The resulting entry vector was used to clone the genes into the destination vector $\mathrm{pK} 7 \mathrm{~m} 34 \mathrm{GW}$, under the control of the $35 \mathrm{~S}$ promoter, and N-terminally fused with GFP with the LR Clonase II Plus enzyme mix (Invitrogen). For the construction of the catalytic site mutant, pDONR P2R-P3-PrKAI2d3 was 
487

488

489

490

491

492

493

494

495

496

497

498

499

500

501

502

503

504

505

506

507

508

509

510

511

512

513

515

514 final volume was adjusted to $100 \mu \mathrm{L}$ with the incubation solution. Plates were incubated 516 illumination at a quantum irradiance of $10 \mu \mathrm{mol} \mathrm{m}^{-2} \mathrm{~s}^{-1}$ (ref. ${ }^{20,26}$ ). A seed was considered 517 germinated when the radicle protruded from the seed coat.

mutated with the QuikChange II site directed mutagenesis kit (Agilent). The generated clones were checked by sequencing. All primers used for cloning are listed (Supplementary Table 4).

Western Blot. Total protein was extracted from 5-day-old seedlings, exposed to white light for $3 \mathrm{~h}$, transferred to darkness for $21 \mathrm{~h}$, and exposed to continuous red light for 4 days. Protein concentrations were determined by the Bradford assay (Bio-Rad). Of the protein extracts, separated by sodium dodecyl sulfate-polyacrylamide gel electrophoresis (SDSPAGE) and transferred onto polyvinylidene fluoride (PVDF) membranes, $30 \mu \mathrm{g}$ was detected with horseradish peroxidase (HRP)-conjugated antibodies against GFP (Anti-GFP-HRP, 1:10000, Miltenyi Biotec) or anti-tubulin (mouse monoclonal, 1/10000, Sigma-Aldrich) and HRP-conjugated anti-mouse antibodies (rabbit polyclonal, 1/10000, Abcam). The blots were visualized with the Western Lightning Plus Enhanced Chemiluminescence kit (PerkinElmer) and the X-Doc System (Bio-Rad). The Precision Plus Protein ${ }^{\mathrm{TM}}$ Dual Color Standards (BioRad) was used as protein size marker.

Arabidopsis hypocotyl elongation assays. Arabidopsis seeds were surface sterilized by consecutive treatments of $5 \mathrm{~min} 70 \%(\mathrm{v} / \mathrm{v})$ ethanol with $0.05 \%(\mathrm{w} / \mathrm{v})$ SDS and $5 \mathrm{~min} 95 \%$ $(\mathrm{v} / \mathrm{v})$ ethanol and sown on half-strength Murashige and Skoog $(1 / 2 \mathrm{MS})$ media (Duchefa Biochemie) containing $1 \%(\mathrm{w} / \mathrm{v})$ agar, supplemented with $1 \mu \mathrm{M}( \pm)-\mathrm{GR} 24[0.01 \%(\mathrm{v} / \mathrm{v})$ DMSO] or with $0.01 \%(\mathrm{v} / \mathrm{v})$ DMSO only (control). Seeds were stratified at $4{ }^{\circ} \mathrm{C}$ for 2 days in the dark, then exposed to white light for $3 \mathrm{~h}$, transferred to darkness for $21 \mathrm{~h}$, and exposed to continuous red light for 4 days at $21^{\circ} \mathrm{C}$. Plates were photographed and hypocotyl lengths were quantified using ImageJ (http://imagej.nih.gov/ij/).

Arabidopsis germination assays. Arabidopsis seeds were after-ripened for at least 6 weeks before use. Surface-sterilized seeds were incubated in the incubation solution (1 mM HEPES buffer; $\mathrm{pH} 7.5)$ at a ratio of $10 \mathrm{mg}$ of seeds per $\mathrm{mL}^{35}$. Fifty $\mu \mathrm{L}$ of seeds $(\sim 20-25$ seeds) were distributed on 96-well plates and $10 \mu \mathrm{L}$ of germination stimulants [(10 $\mu \mathrm{M}(+)-\mathrm{GR} 24,10 \mu \mathrm{M}$ (-)-GR24, and 0.1\% (v/v) DMSO (control) or 10-fold concentrated D-OH] were added. The 15 either for 5 days at $25{ }^{\circ} \mathrm{C}$ in the dark ${ }^{62}$ or for 7 days at $32{ }^{\circ} \mathrm{C}-34{ }^{\circ} \mathrm{C}$ under constant light 
518 Germination stimulation activity assay on root-parasitic plant seeds. Germination stimulant activity of chemicals on seeds of parasitic plants were determined as described previously ${ }^{29}$. Chemicals were suspended in DMSO at $10 \mathrm{mmol} \mathrm{L}^{-1}$, diluted with water at $1 \mathrm{mmol} \mathrm{L}{ }^{-1}$ (water/DMSO; v/v; 9/1), and then of $1 \times 10^{-5} \mathrm{~mol} \mathrm{~L}^{-1}$ to $1 \times 10^{-12} \mathrm{~mol} \mathrm{~L}^{-1}$ with water/DMSO (v/v; 9/1). For each compound, a concentration range from $10^{-13}$ to $10^{-6} \mathrm{~mol} \mathrm{~L}^{-1}$ (water/DMSO; 99/1) was applied to the conditioned parasitic seeds. As negative control, 1\% $(\mathrm{v} / \mathrm{v})$ DMSO was used (seed germination $<1 \%$ ) and as positive control $( \pm)$-GR24 at a concentration of $1 \mu \mathrm{mol} \mathrm{L}{ }^{-1}$, inducing $72-87 \%$ and $50-65 \%$ of seed germination for $P$. ramosa 1 and $S$. hermonthica, respectively. To avoid variations related to sterilization events, the germination percentages are reported as a ratio relative to the positive control $\left[1 \mu \mathrm{mol} \mathrm{L}{ }^{-1}( \pm)\right.$ GR24) included in each germination assay. Each dilution and germination assay were repeated at least three times. For each compound tested, dose response curves (germination stimulation activity $=\mathrm{f}(\mathrm{c})$; germination stimulant activity relative to $1 \mu \mathrm{mol} \mathrm{L}{ }^{-1}( \pm)$-GR24; c, concentration $\left.(\mathrm{mol} \mathrm{L})^{-1}\right)$; half maximal effective concentration $\left(\mathrm{EC}_{50}\right)$; and maximum germination stimulant activity) were determined with a Four Parameter Logistic Curve computed with SigmaPlot ${ }^{\circledR} 10.0$.

Enzymatic degradation of GR24 isomers by purified proteins. The ligand $(10 \mu \mathrm{M})$ was incubated without and with purified AtD14/AtKAI2/PrKAI2d3/PrKAI2d3 ${ }^{\mathrm{S} 98 \mathrm{~A}}(5 \mu \mathrm{M})$ for $150 \mathrm{~min}$ at $25^{\circ} \mathrm{C}$ in $0.1 \mathrm{~mL}$ phosphate buffered saline (PBS; $100 \mathrm{mM}$ Phosphate, $\mathrm{pH} 6.8$, $150 \mathrm{mM} \mathrm{NaCl})$ in the presence of $( \pm)$-1-indanol $(100 \mathrm{M})$ as internal standard. The solutions were acidified to $\mathrm{pH}=1$ by addition of $2 \mu \mathrm{L}$ trifluoroacetic acid (TFA) to quench the reaction and centrifugated (12 $\mathrm{min}, 12,000 \mathrm{tr} / \mathrm{min})$. Thereafter, the samples were subjected to reversephase-ultra-performance liquid chromatography (RP-UPLC)-MS analyses by means of UPLC system equipped with a Photo Diode Array (PDA) and a Triple Quadrupole Detector (TQD) mass spectrometer (Acquity UPLC-TQD, Waters). RP-UPLC (HSS $\mathrm{C}_{18}$ column, $1.8 \mu \mathrm{m}, 2.1$ $\mathrm{mm} \times 50 \mathrm{~mm}$ ) with $0.1 \%(\mathrm{v} / \mathrm{v})$ formic acid in $\mathrm{CH}_{3} \mathrm{CN}$ and $0.1 \%(\mathrm{v} / \mathrm{v})$ formic acid in water (aq. FA, $0.1 \%$, v/v, $\mathrm{pH} 2.8$ ) as eluents $\left[10 \% \mathrm{CH}_{3} \mathrm{CN}\right.$, followed by linear gradient from $10 \%$ to $100 \%$ of $\mathrm{CH}_{3} \mathrm{CN}$ (4 min)] at a flow rate of $0.6 \mathrm{~mL} \mathrm{~min}^{-1}$. The detection was done by PDA and with the TQD mass spectrometer operated in Electrospray ionization-positive mode at $3.2 \mathrm{kV}$ capillary voltage. To maximize the signal, the cone voltage and collision energy were optimized to $20 \mathrm{~V}$ and $12 \mathrm{eV}$, respectively. The collision gas was argon at a pressure maintained near $4.510^{-3} \mathrm{mBar}$. 
550 Enzymatic assays with profluorescent probes and $\boldsymbol{p}$-nitrophenyl acetate. The assays were done as described ${ }^{13}$ with a TriStar LB 941 Multimode Microplate Reader (Berthold Technologies).

Protein melting temperatures. For the Differential Scanning Fluorimetry (DSF) experiments a CFX96 Touch $^{\mathrm{TM}}$ Real-Time PCR Detection System (Bio-Rad) was used with excitation and emission wavelengths of 490 and $575 \mathrm{~nm}$, respectively and Sypro Orange $\left(\lambda_{\text {ex }} / \lambda_{\text {em }}: 470 / 570 \mathrm{~nm}\right.$; Life Technologies) as the reporter dye. Samples were heat-denatured with a linear $25{ }^{\circ} \mathrm{C}$ to $95{ }^{\circ} \mathrm{C}$ gradient at a rate of $1.3{ }^{\circ} \mathrm{C}$ per min after incubation at $25{ }^{\circ} \mathrm{C}$ for 30 min in the dark. The denaturation curve was obtained by means of the CFX manager ${ }^{\mathrm{TM}}$ software. Final reaction mixtures were prepared in triplicate in 96-well white microplates.

560 Each reaction was carried out in $20-\mu \mathrm{L}$ sample in PBS (100 mM phosphate, pH 6.8, $150 \mathrm{mM}$ $\mathrm{NaCl}$ ) containing $6 \mu \mathrm{g}$ of protein (so that the final reactions contained $10 \mu \mathrm{M}$ protein), 0 to $\mathrm{X}$ ligand concentrations in $\mu \mathrm{M}, 4 \%$ (v/v) DMSO, and $0.008 \mu \mathrm{L}$ Sypro Orange. Plates were incubated in the dark for $30 \mathrm{~min}$ before analysis. In the control reaction, DMSO was added instead of the chemical solution. The experiments were repeated three times.

nanoDSF. Proteins were diluted in PBS (100 mM Phosphate, $\mathrm{pH} 6.8,150 \mathrm{mM} \mathrm{NaCl})$ to a concentration of $\sim 10 \mu \mathrm{M}$. Ligands were tested at the concentration of $200 \mu \mathrm{M}$. The intrinsic fluorescence signal was measured as a function of increasing temperature with a Prometheus NT.48 fluorimeter (NanoTemper Technologies), with 55\% excitation light intensity and 1 ${ }^{\circ} \mathrm{C} / \mathrm{min}$ temperature ramp. Analyses were done on capillaries filled with $10 \mu \mathrm{L}$ of the respective samples. Intrinsic fluorescence signals expressed by the $350 \mathrm{~nm} / 330 \mathrm{~nm}$ emission ratio that increases as the proteins unfold, were plotted as a function of temperature (Figures 2d, 2f, and 4d). The plots show one of the three independent data collections done for each protein.

Intrinsic tryptophan fluorescence assays and determination of the dissociation constant

$575 \boldsymbol{K}_{\mathbf{D}}$. The experiments were done as described ${ }^{13}$ by means of the Spark ${ }^{\circledR}$ Multimode Microplate Reader (Tecan).

577 Direct electrospray ionization (ESI)-MS under denaturing conditions. Mass spectrometry measurements were carried out with an electrospray quadrupole-time of flight (Q-TOF) mass spectrometer (Waters) equipped with the Nanomate device (Advion). The HD_A_384 chip

580 ( $5 \mu \mathrm{m}$ i.d. nozzle chip, flow rate range $100-500 \mathrm{~nL} / \mathrm{min}$ ) was calibrated before use. For the 
ESI-MS measurements, the Q-TOF instrument was operated in radio frequency quadrupole mode with the TOF data collected between $m / z$ 400-2990. The collision energy was set to $10 \mathrm{eV}$ and argon was used as collision gas. Mass spectra were acquired after denaturation of $\operatorname{PrKAI} 2 \mathrm{~d} 3 \pm$ ligand in $50 \%(\mathrm{v} / \mathrm{v})$ acetonitrile and 1\% (v/v) formic acid. The Mass Lynx 4.1 (Waters) and Peakview 2.2 (AB Sciex) softwares were used for data acquisition and processing, respectively. Multiply-charged ions were deconvoluted by the MaxEnt algorithm (AB Sciex). The protein average masses were annotated in the spectra and the estimated mass accuracy was $\pm 2 \mathrm{Da}$. For the external calibration, NaI clusters $\left(2 \mu \mathrm{g} / \mu \mathrm{L}\right.$, isopropanol $/ \mathrm{H}_{2} \mathrm{O}$ $50 / 50$, Waters) were used in the acquisition $\mathrm{m} / \mathrm{z}$ mass range.

Localization of the fixation site of ligands on PrKAI2d3. PrKAI2d3-ligand mixtures were incubated for $10 \mathrm{~min}$ prior overnight Glu-C proteolysis. Glu-C--generated peptide mixtures were analyzed by nanoLC-MS/MS with the Triple-TOF 4600 mass spectrometer (AB Sciex) coupled to the nanoRSLC UPLC system (Thermo Fisher Scientific) equipped with a trap column (Acclaim PepMap $100 \mathrm{C}_{18}, 75 \mu \mathrm{m}$ i.d. $\times 2 \mathrm{~cm}, 3 \mu \mathrm{m}$ ) and an analytical column (Acclaim PepMap RSLC C ${ }_{18}, 75 \mu \mathrm{m}$ i.d. $\times 25 \mathrm{~cm}, 2 \mu \mathrm{m}, 100 \AA$ ). Peptides were loaded at $5 \mu \mathrm{L} / \mathrm{min}$ with $0.05 \%(\mathrm{v} / \mathrm{v})$ TFA in $5 \%(\mathrm{v} / \mathrm{v})$ acetonitrile and separated at a flow rate of $300 \mathrm{~nL} / \mathrm{min}$ with a $5 \%$ to $35 \%$ solvent B [0.1\% (v/v) formic acid in $100 \%$ acetonitrile] gradient in $40 \mathrm{~min}$ with solvent A $[0.1 \%(\mathrm{v} / \mathrm{v})$ formic acid in water]. NanoLC-MS/MS experiments were conducted in a Data-Dependent acquisition method by selecting the 20 most intense precursors for collision-induced dissociation fragmentation with the Q1 quadrupole set at a low resolution for better sensitivity. Raw data were processed with the MS Data Converter tool (AB Sciex) for generation of .mgf data files and proteins were identified with the MASCOT search engine (Matrix Science) against the PrKAI2 sequence with oxidation of methionines and ligand-histidine adduct as variable modifications. Peptide and fragment tolerance were set at $20 \mathrm{ppm}$ and $0.05 \mathrm{Da}$, respectively. Only peptides were considered with a MASCOT ion score above the identity threshold (25) calculated at $1 \%$ false discovery rate.

610 Phylogenetic analysis. Phylogenetic analyses were done on 32 D14 and KAI2 sequences, 611 containing five sequences of Phelipanche ramosa obtained in this study and previously 612 described sequences from Phelipanche aegyptiaca, Striga hermonthica, Pisum sativum, 613 Arabidopsis thaliana, and Oryza sativa ${ }^{13,17,19,20,63,64}$. The D14 and KAI2 proteins and the 614 nucleotide data (Supplementary Data files S1 and S2, respectively) were aligned with AFFT ${ }^{65}$ 
615 and the G-INS-i iterative refinement alignment method ${ }^{66}$. Sequence alignments

616 (Supplementary Data files S3 and S4) were manually trimmed to remove gaps at either end, 617 producing final protein and nucleotide data sets of 262-264 amino acids and 786-792 618 nucleotides, respectively (Supplementary Data files S5 and S6). The $\alpha / \beta$ hydrolase RbsQ from 619 Bacillus subtilis was used as outgroup, because of its high similarity to KAI2 and D14, and a 620 conserved catalytic triad ${ }^{17}$. Maximum likelihood (ML) analyses were conducted with $621 \mathrm{RAxML}^{67}$ on 1,000 bootstraps replicates for statistical support of our inferences. The best ML 622 tree for the amino acid sequences was inferred with the PROT-GAMMA model and the WAG 623 substitution matrix. For the nucleotides sequences, the best ML tree was searched with the 624 GTR model and a gamma rate of heterogeneity among sites. The percentage of trees in which 625 the associated taxa clustered together was provided next to the branches. The resulting 626 consensus amino acid and nucleotide trees were drawn to scale with MEGA7 (ref. ${ }^{68}$ ), with 627 the branch lengths representing substitutions per sites.

Modeling. Protein sequences of the $P$. ramosa KAI2 proteins were modeled by means of the SWISSMODEL server (http://swissmodel.expasy.org/) ${ }^{69}$. Models were generated with the chain A of the Apo form of the Arabidopsis KAI2 structure (PDB: 4JYP) as a template ${ }^{70}$. Figures of protein structure were generated with PYMOL. Cavities within homology models were visualized by the surface mode on the setting "cavities and pockets culled" within PYMOL. Pocket sizes were calculated with the CASTp 3.0 server $^{71}$ and a probe radius of $1.4 \AA$. The reported pocket sizes were the Connolly' solvent excluded surface volumes of the catalytic pocket.

Expression data. For the complemented Arabidopsis lines, seeds were imbibed for $24 \mathrm{~h}$ in a growth solution (1 mM HEPES buffer, $\mathrm{pH} 7.5,0.1 \mathrm{mM}$ Preservative Plant Mixture in sterile water) and treated for $6 \mathrm{~h}$ with $10 \mu \mathrm{M}( \pm)$-GR24 or mock solution. P. ramosa seeds were imbibed in the growth solution for 7 days and then treated for $1 \mathrm{~h}$ with $1 \mathrm{nM}( \pm)$-GR2 4 or mock solution.. Seeds were harvested, snap-frozen in liquid nitrogen, and ground with pestle and mortar to a fine powder. RNA was extracted and purified with the RNeasy Plant mini kit

644 (Qiagen). Genomic DNA was removed by DNase treatment and the samples were purified by ammonium acetate $(5 \mathrm{M}$ final concentration) precipitation. The iScript cDNA synthesis kit

646 (Bio-Rad) was used to reverse transcribe RNA. SYBR Green detection was used during qRT647 PCR run on a Light Cycler 480 (Roche). Reactions were done in triplicate in a 384-multiwell 648 plate, in a total volume of $5 \mu \mathrm{L}$ and a cDNA fraction of $10 \%$. Cycle threshold values were 
649 obtained and analyzed with the $2-\Delta \Delta \mathrm{CT}$ method $^{72}$. The values from three biological and three 650 technical repeats were normalized against those of the seed-specific housekeeping gene At4g12590 for Arabidopsis ${ }^{73}$. For P. ramosa, PrCACS was used to normalize the expressions.

652 The normalized values were analyzed according to the published model ${ }^{74}$ with the mixed model procedure (SAS Enterprise).

654

Statistical analyses. As deviations from normality had been observed for the axillary bud length, hypocotyl length, and germination after SL treatments, the Kruskal-Wallis test was used to assess the significance of one treatment with one compound in comparison to a treatment with another by means of the R Commander software (version 1.7-3) (ref. ${ }^{75}$ ).

\section{References}

6601 Parker, C. Parasitic Weeds: A World Challenge. Weed Sci. 60, 269-276,

661 doi:10.1614/WS-D-11-00068.1 (2012).

662

2 Delavault, P., Montiel, G., Brun, G., Pouvreau, J. B., Thoiron, S. \& Simier, P.

663

664

665 Communication Between Host Plants and Parasitic Plants. Adv. Bot. Res. 82, 55-82, doi:10.1016/bs.abr.2016.10.006 (2017).

666

667

668

4 Auger, B., Pouvreau, J.-B., Pouponneau, K., Yoneyama, K., Montiel, G., Le Bizec, B., germination in parasitic plants: what insights can we expect from strigolactone research? J. Exp. Bot. 69, 2265-2280, doi:10.1093/jxb/erx472 (2017). Yoneyama, K., Delavault, P., Delourme, R. \& Simier, P. Germination Stimulants of Phelipanche ramosa in the Rhizosphere of Brassica napus Are Derived from the Glucosinolate Pathway. Mol. Plant-Microbe Interact. 25, 993-1004, doi:10.1094/mpmi-01-12-0006-r (2012). parasitic weed Orobanche crenata. Agric. Ecosyst. Environ. 122, 275-281, doi:10.1016/j.agee.2007.01.014 (2007). Underground Mechanisms of Parasitism and Associated Strategies for their Control: A Review. Front. Plant Sci. 7, 135, doi:10.3389/fpls.2016.00135 (2016). 
8 Brundrett, M. C. \& Tedersoo, L. Evolutionary history of mycorrhizal symbioses and global host plant diversity. New Phytol. 220, 1108-1115, doi:10.1111/nph.14976 (2018).

9 Lopez-Obando, M., Ligerot, Y., Bonhomme, S., Boyer, F.-D. \& Rameau, C. Strigolactone biosynthesis and signaling in plant development. Development 142, 3615-3619, doi:10.1242/dev.120006 (2015).

10 Hamiaux, C., Drummond, R. S. M., Janssen, B. J., Ledger, S. E., Cooney, J. M., Newcomb, R. D. \& Snowden, K. C. DAD2 Is an alpha/beta Hydrolase Likely to Be Involved in the Perception of the Plant Branching Hormone, Strigolactone. Curr. Biol. 22, 2032-2036, doi:10.1016/j.cub.2012.08.007 (2012).

11 Waters, M. T., Gutjahr, C., Bennett, T. \& Nelson, D. C. Strigolactone Signaling and Evolution. Annu. Rev. Plant Biol. 68, 291-322, doi:10.1146/annurev-arplant-042916040925 (2017).

12 Nakamura, H., Xue, Y. L., Miyakawa, T., Hou, F., Qin, H. M., Fukui, K., Shi, X., Ito, E., Ito, S., Park, S. H., Miyauchi, Y., Asano, A., Totsuka, N., Ueda, T., Tanokura, M. \& Asami, T. Molecular mechanism of strigolactone perception by DWARF14. Nat. Commun. 4, 2613, doi:10.1038/ncomms3613 (2013).

13 de Saint Germain, A., Clavé, G., Badet-Denisot, M.-A., Pillot, J.-P., Cornu, D., Le Caer, J.-P., Burger, M., Pelissier, F., Retailleau, P., Turnbull, C., Bonhomme, S., Chory, J., Rameau, C. \& Boyer, F.-D. An histidine covalent receptor and butenolide complex mediates strigolactone perception. Nat. Chem. Biol. 12, 787-794, doi:10.1038/nchembio.2147 (2016).

14 Yao, R., Ming, Z., Yan, L., Li, S., Wang, F., Ma, S., Yu, C., Yang, M., Chen, L., Li, Y., Yan, C., Miao, D., Sun, Z., Yan, J., Sun, Y., Wang, L., Chu, J., Fan, S., He, W., Deng, H., Nan, F., Li, J., Rao, Z., Lou, Z. \& Xie, D. DWARF14 is a non-canonical hormone receptor for strigolactone. Nature 536, 469-473, doi:10.1038/nature19073 (2016).

15 Shabek, N., Ticchiarelli, F., Mao, H., Hinds, T. R., Leyser, O. \& Zheng, N. Structural plasticity of D3-D14 ubiquitin ligase in strigolactone signalling. Nature 563, 652-656, doi:10.1038/s41586-018-0743-5 (2018).

16 Seto, Y., Yasui, R., Kameoka, H., Tamiru, M., Cao, M., Terauchi, R., Sakurada, A., Hirano, R., Kisugi, T., Hanada, A., Umehara, M., Seo, E., Akiyama, K., Burke, J., Takeda-Kamiya, N., Li, W., Hirano, Y., Hakoshima, T., Mashiguchi, K., Noel, J. P., Kyozuka, J. \& Yamaguchi, S. Strigolactone perception and deactivation by a hydrolase receptor DWARF14. Nat. Commun. 10, 191, doi:10.1038/s41467-01808124-7 (2019).

17 Waters, M. T., Nelson, D. C., Scaffidi, A., Flematti, G. R., Sun, Y. K., Dixon, K. W. \& Smith, S. M. Specialisation within the DWARF14 protein family confers distinct responses to karrikins and strigolactones in Arabidopsis. Development 139, 12851295, doi:10.1242/dev.074567 (2012). 
Swarbreck, S. M., Guerringue, Y., Matthus, E., Jamieson, F. J. C. \& Davies, J. M. Impairment in karrikin but not strigolactone sensing enhances root skewing in Arabidopsis thaliana. Plant J. 98, 607-621, doi:10.1111/tpj.14233 (2019).

Conn, C. E., Bythell-Douglas, R., Neumann, D., Yoshida, S., Whittington, B., Westwood, J. H., Shirasu, K., Bond, C. S., Dyer, K. A. \& Nelson, D. C. Convergent evolution of strigolactone perception enabled host detection in parasitic plants. Science 349, 540-543, doi:10.1126/science.aab1140 (2015).

Toh, S., Holbrook-Smith, D., Stogios, P. J., Onopriyenko, O., Lumba, S., Tsuchiya, Y., Savchenko, A. \& McCourt, P. Structure-function analysis identifies highly sensitive strigolactone receptors in Striga. Science 350, 203-207, doi:10.1126/science.aac9476 (2015).

21 Yao, R., Wang, F., Ming, Z. H., Du, X. X., Chen, L., Wang, Y. P., Zhang, W. H., Deng, H. T. \& Xie, D. X. ShHTL7 is a non-canonical receptor for strigolactones in root parasitic weeds. Cell Res. 27, 838-841, doi:10.1038/cr.2017.3 (2017).

Goyet, V., Billard, E., Pouvreau, J.-B., Lechat, M.-M., Pelletier, S., Bahut, M., in the obligate parasitic plant Phelipanche ramosa involves a host-exudated cytokinin

23 Yang, Z., Zhang, Y., Wafula, E. K., Honaas, L. A., Ralph, P. E., Jones, S., Clarke, C. R., Liu, S., Su, C., Zhang, H., Altman, N. S., Schuster, S. C., Timko, M. P., Yoder, J. I., Westwood, J. H. \& dePamphilis, C. W. Horizontal gene transfer is more frequent with increased heterotrophy and contributes to parasite adaptation. Proc. Natl. Acad. Sci. U.S.A. 113, E7010, doi:10.1073/pnas.1608765113 (2016). signal. J. Exp. Bot. 68, 5539-5552, doi:10.1093/jxb/erx359 (2017).

Wicke, S., Müller, K. F., dePamphilis, C. W., Quandt, D., Bellot, S. \& Schneeweiss, G. M. Mechanistic model of evolutionary rate variation en route to a nonphotosynthetic lifestyle in plants. Proc. Natl. Acad. Sci. U.S.A. 113, 9045, doi:10.1073/pnas.1607576113 (2016).

Fernández-Aparicio, M., Rubiales, D., Bandaranayake, P. C. G., Yoder, J. I. \& Westwood, J. H. Transformation and regeneration of the holoparasitic plant Phelipanche aegyptiaca. Plant Methods 7, 36, doi:10.1186/1746-4811-7-36 (2011).

Toh, S., Kamiya, Y., Kawakami, N., Nambara, E., McCourt, P. \& Tsuchiya, Y. Thermoinhibition uncovers a role for strigolactones in Arabidopsis seed germination. Plant Cell Physiol. 53, 107-117, doi:10.1093/pcp/pcr176 (2012).

27 Boyer, F.-D., de Saint Germain, A., Pillot, J.-P., Pouvreau, J.-B., Chen, V. X., Ramos, 1524-1544, doi:10.1104/pp.112.195826 (2012). 
A., Stevens, C. V., Geelen, D., Goormachtig, S. \& Rameau, C. New Strigolactone Analogs as Plant Hormones with Low Activities in the Rhizosphere. Mol. Plant 7, 675-690, doi:10.1093/mp/sst163 (2014).

29 Pouvreau, J.-B., Gaudin, Z., Auger, B., Lechat, M. M., Gauthier, M., Delavault, P. \& Simier, P. A high-throughput seed germination assay for root parasitic plants. Plant methods 9, 32, doi:10.1186/1746-4811-9-32 (2013).

30 Tsuchiya, Y., Yoshimura, M., Sato, Y., Kuwata, K., Toh, S., Holbrook-Smith, D., Zhang, H., McCourt, P., Itami, K., Kinoshita, T. \& Hagihara, S. Probing strigolactone receptors in Striga hermonthica with fluorescence. Science 349, 864-868, doi:10.1126/science.aab3831 (2015).

31 Yao, R., Wang, L., Li, Y., Chen, L., Li, S., Du, X., Wang, B., Yan, J., Li, J. \& Xie, D. Rice DWARF14 acts as an unconventional hormone receptor for strigolactone. J. Exp. Bot. 69, 2355-2365, doi:10.1093/jxb/ery014 (2018).

32 Satchell, D. P. N., Satchell, R. S. \& Wassef, W. N. The Kinetics and Mechanism of Addition of Water and Alcohols to $p$-Nitrophenyl Isothiocyanate. The Effects of Added Dimethyl Sulfoxide. Z. Naturforsch., B: Chem. Sci. 45, 1032-1036, doi:10.1515/znb-1990-0721 (1990).

33 Waters, M. T. Spoilt for Choice: New Options for Inhibitors of Strigolactone Signaling. Mol. Plant 12, 21-23, doi:10.1016/j.molp.2018.11.012 (2019).

34 Zehhar, N., Ingouff, M., Bouya, D. \& Fer, A. Possible involvement of gibberellins and ethylene in Orobanche ramosa germination. Weed Res. 42, 464-469, doi:10.1046/j.1365-3180.2002.00306.x (2002).

35 Lechat, M.-M., Brun, G., Montiel, G., Veronesi, C., Simier, P., Thoiron, S., Pouvreau, J.-B. \& Delavault, P. Seed response to strigolactone is controlled by abscisic acidindependent DNA methylation in the obligate root parasitic plant, Phelipanche ramosa L. Pomel. J. Exp. Bot. 66, 3129-3140, doi:10.1093/jxb/erv119 (2015).

36 Takahashi, I. \& Asami, T. Target-based selectivity of strigolactone agonists and antagonists in plants and their potential use in agriculture. J. Exp. Bot. 69, 2241-2254, doi:10.1093/jxb/ery126 (2018).

37 Pepperman, A. B., Connick, W. J., Vail, S. L., Worsham, A. D., Pavlista, A. D. \& Moreland, D. E. Evaluation of Precursors and Analogs of Strigol as Witchweed (Striga asiatica) Seed Germination Stimulants. Weed Sci. 30, 561-566, doi:10.1017/S0043174500041175 (1982).

38 Johnson, A. W., Rosebery, G. \& Parker, C. A novel approach to Striga and Orobanche control using synthetic germination stimulants. Weed Res. 16, 223-227, doi:10.1111/j.1365-3180.1976.tb00406.x (1976).

39 Xu, Y., Miyakawa, T., Nosaki, S., Nakamura, A., Lyu, Y., Nakamura, H., Ohto, U., Ishida, H., Shimizu, T., Asami, T. \& Tanokura, M. Structural analysis of HTL and 
D14 proteins reveals the basis for ligand selectivity in Striga. Nat. Commun. 9, 3947, doi:10.1038/s41467-018-06452-2 (2018).

40 Vurro, M., Prandi, C. \& Baroccio, F. Strigolactones: how far is their commercial use for agricultural purposes? Pest. Manage. Sci. 72, 2026-2034, doi:10.1002/ps.4254 (2016).

41 Lee, H. W., Sharma, P., Janssen, B. J., Drummond, R. S. M., Luo, Z., Hamiaux, C., Collier, T., Allison, J. R., Newcomb, R. D. \& Snowden, K. C. Flexibility of the petunia strigolactone receptor DAD2 promotes its interaction with signaling partners. J. Biol. Chem., doi:10.1074/jbc.RA119.011509 (2020).

42 Yao, J., Mashiguchi, K., Scaffidi, A., Akatsu, T., Melville, K. T., Morita, R., Morimoto, Y., Smith, S. M., Seto, Y., Flematti, G. R., Yamaguchi, S. \& Waters, M. T. An allelic series at the KARRIKIN INSENSITIVE 2 locus of Arabidopsis thaliana decouples ligand hydrolysis and receptor degradation from downstream signalling. Plant J. 96, 75-89, doi:10.1111/tpj.14017 (2018).

43 Jamil, M., Kountche, B. A., Haider, I., Wang, J. Y., Aldossary, F., Zarban, R. A., Jia, K. P., Yonli, D., Shahul Hameed, U. F., Takahashi, I., Ota, T., Arold, S. T., Asami, T. \& Al-Babili, S. Methylation at the C-3' in D-Ring of Strigolactone Analogs Reduces Biological Activity in Root Parasitic Plants and Rice. Front. Plant Sci. 10, 353, doi:10.3389/fpls.2019.00353 (2019).

44 Yamauchi, M., Ueno, K., Furumoto, T., Wakabayashi, T., Mizutani, M., Takikawa, H. \& Sugimoto, Y. Stereospecific reduction of the butenolide in strigolactones in plants. Bioorg. Med. Chem. 26, 4225-4233, doi:10.1016/j.bmc.2018.07.016 (2018).

45 Zwanenburg, B. \& Pospíšil, T. Structure and Activity of Strigolactones: New Plant Hormones with a Rich Future. Mol. Plant 6, 38-62, doi:10.1093/mp/sss141 (2013).

46 Uraguchi, D., Kuwata, K., Hijikata, Y., Yamaguchi, R., Imaizumi, H., Am, S., Rakers, C., Mori, N., Akiyama, K., Irle, S., McCourt, P., Kinoshita, T., Ooi, T. \& Tsuchiya, Y. A femtomolar-range suicide germination stimulant for the parasitic plant Striga hermonthica. Science 362, 1301, doi:10.1126/science.aau5445 (2018).

47 Johnson, A. W., Gowda, G., Hassanali, A., Knox, J., Monaco, S., Razavi, Z. \& Rosebery, G. The Preparation of Synthetic Analogs of Strigol. J. Chem. Soc., Perkin Trans. 1, 1734-1743, doi:10.1039/P19810001734 (1981).

48 Yoneyama, K. Small-Molecule inhibitors: Weed-control measures. Nat. Chem. Biol. 12, 658-659, doi:10.1038/nchembio.2155 (2016).

49 Pickett, J. A., Wadhams, L. J. \& Woodcock, C. M. Developing sustainable pest control from chemical ecology. Agric. Ecosyst. Environ. 64, 149-156, doi:10.1016/s0167-8809(97)00033-9 (1997).

50 Mangnus, E. M., Dommerholt, F. J., Dejong, R. L. P. \& Zwanenburg, B. Improved Synthesis of Strigol Analog GR24 and Evaluation of the Biological-Activity of Its 

(1992).

51 de Saint Germain, A., Retailleau, P., Norsikian, S., Servajean, V., Pelissier, F., Steinmetz, V., Pillot, J.-P., Rochange, S., Pouvreau, J.-B. \& Boyer, F.-D. Contalactone, a contaminant formed during chemical synthesis of the strigolactone reference GR24 is also a strigolactone mimic. Phytochemistry 168, 112112, doi:10.1016/j.phytochem.2019.112112 (2019).

Hamiaux, C., Drummond, R. S. M., Luo, Z. W., Lee, H. W., Sharma, P., Janssen, B. J., Perry, N. B., Denny, W. A. \& Snowden, K. C. Inhibition of strigolactone receptors by N-phenylanthranilic acid derivatives: Structural and functional insights. J. Biol. Chem. 293, 6530-6543, doi:10.1074/jbc.RA117.001154 (2018). Kim, D., Al - Babili, S. \& Arold, S. T. Structural basis for specific inhibition of the highly sensitive ShHTL7 receptor. EMBO Rep. 19, e45619, doi:10.15252/embr.201745619 (2018).

54 Nakamura, H., Hirabayashi, K., Miyakawa, T., Kikuzato, K., Hu, W., Xu, Y., Jiang, K., Takahashi, I., Niiyama, R., Dohmae, N., Tanokura, M. \& Asami, T. Triazole Ureas Covalently Bind to Strigolactone Receptor and Antagonize Strigolactone Responses. Mol. Plant 12, 44-58, doi:10.1016/j.molp.2018.10.006 (2019).

Holbrook-Smith, D., Toh, S., Tsuchiya, Y. \& McCourt, P. Small-molecule antagonists of germination of the parasitic plant Striga hermonthica. Nat. Chem. Biol. 12, 724729, doi:10.1038/nchembio.2129 (2016).

Rameau, C., Bodelin C, Cadier D, Grandjean O, Miard F \& Murfet IC. New ramosus mutants at loci Rms $1, R m s 3$ and $R m s 4$ resulting from the mutation breeding program at Versailles. Pisum Genetics 29, 7-12 (1997).

Braun, N., de Saint Germain, A., Pillot, J. P., Boutet-Mercey, S., Dalmais, M., Antoniadi, I., Li, X., Maia-Grondard, A., Le Signor, C., Bouteiller, N., Luo, D., Bendahmane, A., Turnbull, C. \& Rameau, C. The pea TCP transcription factor PsBRC1 acts downstream of Strigolactones to control shoot branching. Plant Physiol. 158, 225-238, doi:10.1104/pp.111.182725 (2012).

Toh, S., Holbrook-Smith, D., Stokes, M. E., Tsuchiya, Y. \& McCourt, P. Detection of Arabidopsis HTL/KAI2 Strigolactone Perception System. Chem. Biol. 21, 988-998, doi:10.1016/j.chembiol.2014.07.005 (2014).

87560 Clough, S. J. \& Bent, A. F. Floral dip: a simplified method for Agrobacterium- 
61 Stojanova, B., Delourme, R., Duffé, P., Delavault, P. \& Simier, P. Genetic differentiation and host preference reveal non-exclusive host races in the generalist parasitic weed Phelipanche ramosa. Weed Res. 59, 107-118, doi:10.1111/wre.12353 (2019).

62 Brun, G., Thoiron, S., Braem, L., Pouvreau, J.-B., Monatiel, G., Lechat, M.-M., Simier, P., Gevaert, K., Goormachtig, S. \& Delavault, P. CYP707As are effectors of karrikin and strigolactone signaling pathways in Arabidopsis thaliana and parasitic plants. Plant Cell Environ. 0, doi:10.1111/pce.13594 (2019).

63 Arite, T., Umehara, M., Ishikawa, S., Hanada, A., Maekawa, M., Yamaguchi, S. \& Kyozuka, J. d14, a Strigolactone-Insensitive Mutant of Rice, Shows an Accelerated Outgrowth of Tillers. Plant Cell Physiol. 50, 1416-1424, doi:10.1093/pcp/pcp091 (2009).

64 Carbonnel, S., Torabi, S., Griesmann, M., Bleek, E., Tang, Y., Buchka, S., Basso, V., Shindo, M., Wang, T. L., Udvardi, M., Waters, M. \& Gutjahr, C. Duplicated KAI2 receptors with divergent ligand-binding specificities control distinct developmental traits in Lotus japonicus. bioRxiv, 754937, doi:10.1101/754937 (2019).

65 Katoh, K., Rozewicki, J. \& Yamada, K. D. MAFFT online service: multiple sequence alignment, interactive sequence choice and visualization. Briefings Bioinf. 20, 11601166, doi:10.1093/bib/bbx108 (2019).

66 Katoh, K., Kuma, K., Toh, H. \& Miyata, T. MAFFT version 5: improvement in accuracy of multiple sequence alignment. Nucleic Acids Res. 33, 511-518, doi:10.1093/nar/gki198 (2005).

67 Stamatakis, A. RAxML version 8: a tool for phylogenetic analysis and post-analysis of large phylogenies. Bioinformatics 30, 1312-1313, doi:10.1093/bioinformatics/btu033 (2014).

68 Kumar, S., Stecher, G. \& Tamura, K. MEGA7: Molecular Evolutionary Genetics Analysis Version 7.0 for Bigger Datasets. Mol. Biol. Evol. 33, 1870-1874, doi:10.1093/molbev/msw054 (2016).

69 Waterhouse, A., Bertoni, M., Bienert, S., Studer, G., Tauriello, G., Gumienny, R., Heer, F. T., de Beer, T. A P., Rempfer, C., Bordoli, L., Lepore, R. \& Schwede, T. SWISS-MODEL: homology modelling of protein structures and complexes. Nucleic Acids Res. 46, W296-W303, doi:10.1093/nar/gky427 (2018).

70 Guo, Y., Zheng, Z., La Clair, J. J., Chory, J. \& Noel, J. P. Smoke-derived karrikin perception by the alpha/beta-hydrolase KAI2 from Arabidopsis. Proc. Natl. Acad. Sci. U.S.A. 110, 8284-8289, doi:10.1073/pnas.1306265110 (2013).

71 Tian, W., Chen, C., Lei, X., Zhao, J. \& Liang, J. CASTp 3.0: computed atlas of surface topography of proteins. Nucleic Acids Res. 46, W363-W367, doi:10.1093/nar/gky473 (2018). 
$91672 \quad$ Livak, K. J. \& Schmittgen, T. D. Analysis of relative gene expression data using realtime quantitative PCR and the 2(T)(-Delta Delta C) method. Methods 25, 402-408, doi:10.1006/meth.2001.1262 (2001).

73 Dekkers, B. J. W., Willems, L., Bassel, G. W., van Bolderen-Veldkamp, R. P.,

74 Rasmussen, A., Mason, M. G., De Cuyper, C., Brewer, P. B., Herold, S., Agusti, J., Geelen, D., Greb, T., Goormachtig, S., Beeckman, T. \& Beveridge, C. A.

924 Strigolactones suppress adventitious rooting in Arabidopsis and pea. Plant Physiol. 158, 1976-1987, doi:10.1104/pp.111.187104 (2012). for RT-qPCR Expression Analysis in Arabidopsis and Tomato Seeds. Plant Cell Physiol. 53, 28-37, doi:10.1093/pcp/pcr113 (2011).

Fox, J. Using the R commander: A point-and-click interface for $\boldsymbol{R}$. (Chapman \& Hall/CRC Press, 2016). Phelipanche ramosa seed germination in response to the strigolactone analogue GR24. J. Exp. Bot. 63, 5311-5322, doi:10.1093/jxb/ers189 (2012).

Aknowledgements. We thank J.-P. Pillot for the pea plant bioassays, Bruno Baron (Institut Pasteur, France) for access to and help with the nanoDSF experiments, Thomas Larribeau for technical assistance, and Catherine Rameau, Sandrine Bonhomme and Martine De Cock for their comments on the manuscript. This work was supported by the Institut Jean-Pierre Bourgin's Plant Observatory technological platforms, a "Infrastructures en Biologie Santé et Agronomie" grant to SICAPS platform of the Institute for Integrative Biology of the Cell, and CHARM3AT Labex program (ANR-11-LABX-39). A.d.S.G. is the recipient of an 942 AgreenSkills award from the European Union in the framework of the Marie-Curie FP7 943 COFUND People Programme and fellowship from Saclay Plant Sciences (ANR-17-EUR944 0007). A.J. is indebted to the Research Foundation-Flanders for a Structural Basic Research 945 fellowship (Project 1S15817N) and for a travel grant in the framework of a Tournesol fellowship (Project VS04418N).

Author contributions. A.d.S.G., J.-B.P., and F.-D.B. designed research; G.C. designed and synthesized the profluorescent probes; V.Se. and F.-D.B. synthesized the chemicals; A.d.S.G.,

949 A.J., and E.B. produced and purified the proteins; A.d.S.G. and A.J. characterized the proteins 950 and did the kinetic experiments; A.J., G.B., J.-B.P., and F.-D.B. performed the plant 
951 experiments; D.C. performed the mass experiments; A.d.S.G., V.St., and F.-D.B. performed 952 the HPLC analyses and separations; A.d.S.G., A.J., G.B., J.-B.P., L.B, D.C., K.G., P.S., S.W., 953 S.G., P.D., and F.-D.B. analyzed the data; A.d.S.G., A.J., G.B., J.-B.P., and F.-D.B. wrote the 954 paper. All authors critically revised the manuscript.

955 Competing interests. The authors declare no competing interests.

\section{Additional information}

957 Supplementary information is available for this paper at www....

958 Materials and Correspondence and requests for materials should be addressed to F.-D.B. 


\section{Legend to Figures}

961

962

963

964

965

966

967

968

969

970

971

972

973

974

975

976

977

978

979

980

981

982

983

984

985

986

987

988

989

990

991

Figure 1. Identification of PrKAI2 putative SL receptors in $P$. ramosa. (a) Phylogenetic analysis of KAI2 and D14 amino acid sequences. The phylogenetic tree was constructed with the maximum likelihood method and 1,000 bootstraps replicates by means of RAxML. The scale bar represents 0.05 substitutions per site. Clades were designated as described ${ }^{19}$ (b) Amino acid sequence alignment of the active PrKAI2 protein sites. Amino acids that differed from AtKAI2 and those similar to AtD14 are in orange and red, respectively; whereas. AtHTL and PrKAI2d3 are in blue and yellow, respectively. A fully expanded alignment can be found in Supplementary Figure 3. (c) Visual representation of the ligand pockets of the $P$. ramosa KAI2 genes. The KAI2 protein sequences were modeled with the chain A of the karrikin-bound Arabidopsis KAI2 structure as a template (PDB: 4JYP). The protein structures were generated with PYMOL and the cavities within the homology models were visualized with the surface mode on the setting "cavities and pockets culled" within PYMOL. (d) Expression of the KAI2 genes in P. ramosa. Primers for PrKAI2c and PrKAI2d 3 were used in a qRT-PCR experiment with EF101 as housekeeping gene on seeds treated for $1 \mathrm{~h}$ with $( \pm)$ GR24 ${ }^{76}(P<0.001$, Student's $t$ test). (e-g) Cross-species complementation assays of the $h t l-3$ mutant with P. ramosa KAI2d3 and the catalytic site mutant S98A. (e) Protein levels of PrKAI2d3 of 4-day-old seedlings transformed with p35S::GFP:PrKAI2d3 or p35S::GFP:PrKAI2 $23^{S 98 A}$ detected with anti-GFP (top) and anti-tubulin (bottom) antibodies as loading control. The experiment was repeated twice with comparable results and one representative repeat is shown. (f) Seed germination after 5 days of growth at $25^{\circ} \mathrm{C}$ in the dark, with DMSO (control), $10 \mu \mathrm{M}(+)$-GR24, or $10 \mu \mathrm{M}(-)$-GR24 treatments. Transgenes were expressed in the null htl-3 mutant background (Col-0 accession) under control of the $35 \mathrm{~S}$ promoter. (one representative experiment of 18 wells per condition with an average of 19 seeds/well shown). (g) Hypocotyl lengths of 4-day-old seedlings grown under continuous red light at $21{ }^{\circ} \mathrm{C}(n=25)$ with $10 \mu \mathrm{M}( \pm)$-GR24 treatments. Graphs represent means of three biological repeats \pm SE. Statistical groups indicated by letters were determined by KruskalWallis test with Dunn's post test, $P<0.001$ (f) and $P<0.05$ (g).

Figure 2. PrKAI2d3 shows a stereoselectivity towards GR24 analogs mimicking the SL natural stereoconfigurations and perceives SL analogs without a methyl group on the Dring. (a) Structures of GR24 analogs. (b) and (c) Germination stimulation activity on $P$. ramosa seeds $\left(\mathrm{EC}_{50}\right)$ with four stereoisomers and with GR24 analogs harboring variation on 
the D-ring ( \pm SE), respectively. (d-g) Thermostability of PrKAI2d3 at $10 \mu \mathrm{M}$ in the absence of ligands (black line) or in the presence of various ligands at $100 \mu \mathrm{M}$ analyzed by nanoDSF.

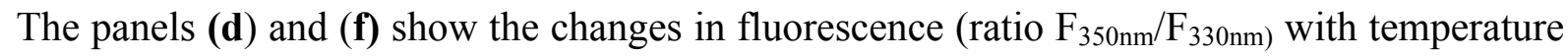
and (e) and (g) the first derivatives for the $\mathrm{F}_{350 \mathrm{~nm}} / \mathrm{F}_{330 \mathrm{~nm}}$ curve against the temperature gradient from which the apparent melting temperatures $\left(\mathrm{T}_{\mathrm{m}}\right)$ was determined for each sample. The experiment was carried out twice. (h) and (i) SL analogs binding PrKAI2d3 based on intrinsic tryptophan fluorescence. Plots of fluorescence intensity versus probe concentrations. The change in intrinsic fluorescence was monitored (Supplementary Figure 8) and used to determine the apparent $K_{\mathrm{D}}$ values. The plots represent the mean of two replicates and the experiments were repeated at least three times. The analysis was done with GraphPad Prism 5.0 Software.

Figure 3. The PrKAId3 enzymatic activity improves the SL biological activity. (a) and (b) Hydrolysis activity of GR24 isomers and analogs by various proteins. (+)-GR24, (-)-GR24, (+)-2'epi-GR24 and (-)-2'epi-GR24 (a) and ( \pm )-GR24, ( \pm )-4'-desmethyl-GR24, ( \pm )-4'-

1006 desmethyl-2'-epi-GR24, and ( \pm )-3'-Me-GR24 (b) at $10 \mu \mathrm{M}$ were incubated with PrKAI2d3, 1007 PrKAI2d3 ${ }^{\text {S98A }}$, AtD14, and AtKAI2 at $5 \mu \mathrm{M}$ for $150 \mathrm{~min}$ at $25^{\circ} \mathrm{C}$. UPLC-UV (260 nm) analysis was used to detect the remaining amount of GR24 isomers and analogs. Bars represent the mean value of the hydrolysis rate calculated from the remaining GR24 isomers and analogs taking into account the hydrolysis in the buffer alone (without protein sample), quantified with indanol as internal standard. Error bars represent the SD of three replicates (means $\pm \mathrm{SD}, n=3$ ). nd, no hydrolysis detected. The asterisks indicate statistical significance

1013 from the PrKAI2d 3 protein sample as $* * * P \leq 0.001$; and $P>0.05$, as measured by Kruskal1014 Wallis test. (c-e) Enzymatic kinetics for PrKAI2d3, PrKAI2d3 ${ }^{\text {S98A }}$, AtD14 and AtKAI2 1015 proteins incubated with $( \pm)-G C 486$ (c), $( \pm)-G C 240$ (d), and ( \pm )-GC242 (e). Progress curves 1016 during hydrolysis of the probes, monitored $\left(\lambda_{\mathrm{em}} 460 \mathrm{~nm}\right)$ at $25^{\circ} \mathrm{C}$ with the use of $400 \mathrm{nM}$ 1017 protein and $20 \mu \mathrm{M}$ probes. The traces represent one of the three replicates and the 1018 experiments were repeated at least twice. (f-h) Hyperbolic plot of the PrKAI2d3 presteady1019 state kinetics reaction velocity with $( \pm)-G C 486(\mathbf{f}),( \pm)-G C 240(\mathbf{g})$, and ( \pm )-GC242 (h). The 1020 initial velocity was determined with profluorescent probe concentrations from $0.3 \mu \mathrm{M}$ to $102140 \mu \mathrm{M}$ and with proteins at $400 \mathrm{nM}$. Error bars represent SE of the mean of three replicates 1022 and the experiments were repeated at least three times. (i) Kinetics constants of probes 1023 towards PrKAI2d3. $K_{1 / 2}$ and $k_{\text {cat }}$ are presteady-state kinetics constants for PrKAI2d3 with different profluorescent probes and represent the mean \pm SE of three replicates. (j-o) Mass 
1025 spectrometry characterization of covalent PrKAI2d3-ligand complexes. On the left, 1026 deconvoluted electrospray mass spectra of PrKAI2d3 prior and after addition of different 1027 ligands $( \pm)$-GR24 and ( \pm )-GC486. Peaks with an asterisk correspond to PrKAI2d3 covalently 1028 bound to a ligand (PrKAI2d3-ligand). The mass increments were measured for different 1029 PrKAI2d3-ligand complexes: 96.3 Da ( \pm )-GR24) and 82.4 Da ( \pm )-GC486). Ligand-modified 1030 amino acids were identified by nanoLC-MSMS analyses after Glu-C proteolysis. On the right, 1031 fragmentation spectra of unmodified and different ligand-modified peptides. Labeled peaks 1032 correspond to the $\mathrm{b}$ and $\mathrm{y}$ fragments of the double-charged precursor ion displayed at the top. 1033 The histidine residue modified by different ligands is underlined.

1034 Figure 4. The isothiocyanate germination stimulants are perceived by PrKAI2d3. (a)

1035 Structure of isothiocyanate. (b) and (c) Modeled curves of dose response germination 1036 stimulant activities (b) and $\mathrm{EC}_{50}$ (half maximal effective concentration) (c). Data presented \pm 1037 SE. (d) and (e) Thermostability of PrKAI2d3 at $10 \mu \mathrm{M}$ in the absence of ligand or in the 1038 presence of various ligands at $100 \mu \mathrm{M}$ analyzed by nanoDSF. Changes in fluorescence (ratio $1039 \mathrm{~F}_{350 \mathrm{~nm}} / \mathrm{F}_{330 \mathrm{~nm}}$ ) with temperature (d) and first derivatives for the $\mathrm{F}_{350 \mathrm{~nm}} / \mathrm{F}_{330 \mathrm{~nm}}$ curve against the 1040 temperature gradient from which the apparent melting temperatures $\left(\mathrm{T}_{\mathrm{m}}\right)$ were determined. for 1041 each sample (e). The experiment was carried out twice. (f-h) Mass spectrometry 1042 characterization of covalent PrKAI2d3-ligand complexes. Deconvoluted electrospray mass 1043 spectra of PrKAI2d3 (f) and PrKAI2d3 ${ }^{\text {S98A }}(\mathrm{g})$ after addition of the 2-PEITC ligand, Peaks 1044 with an asterisk correspond to PrKAI2d3 covalently bound to a ligand (PrKAI2d3-ligand). 1045 The mass increments were measured for the PrKAI2d3-2-PEITC complex, 162.7 Da. Ligand1046 modified amino acids were identified by nanoLC-MSMS analyses after Glu-C proteolysis. (h) 1047 Fragmentation spectra of unmodified and different ligand-modified peptides. Labeled peaks 1048 correspond to the $\mathrm{b}$ and $\mathrm{y}$ fragments of the double-charged precursor ion displayed at the top. 1049 The PEITC ligand-modified serine residue is underlined. (i) Perception mechanism of PEITC.

1050 Figure 5. Identification of antagonists and agonists for the $\boldsymbol{P}$. ramosa seed germination.

1051 (a) Chemical structures of inhibitors of D14 protein and absisic acid (ABA). (b) and (c) 1052 Comparison of the inhibitor effect on P. ramosa germination stimulation (GS) induced by 1053 GR24 $(10 \mathrm{nM})$ or 2-PEITC $(100 \mathrm{nM})$. Maximum of inhibition (b) and $\mathrm{IC}_{50}$ (c). (d) Structure 1054 of D-OH rings and derivatives. (e) and (f) GS activity of D-OH and its derivatives on $P$. 1055 ramosa seeds. Maximum of GS activity (e) and $\mathrm{EC}_{50}(\mathbf{f})$. (g) D-OH binding of PrKAI2d3, 1056 based on intrinsic tryptophan fluorescence. Plots of fluorescence intensity versus probe 
1057 concentrations. The change in intrinsic fluorescence was monitored and used to determine the 1058 apparent $K_{\mathrm{D}}$ values. The plots represent the mean of two replicates and the experiments were 1059 repeated at least three times. The analysis was done with GraphPad Prism 5.0 Software. (h)

1060 Biochemical analysis of the interaction between PrKAI2d 3 at $10 \mu \mathrm{M}$ and D-OH at various 1061 concentrations by DSF. Each line represents the average protein melt curve for two technical 1062 replicates and the experiment was carried out twice. (i) and (j) Mass spectrometry 1063 characterization of covalent PrKAI2d3-D-OH complexes. (i) Deconvoluted electrospray mass 1064 spectrum of PrKAI2d3 after addition of the D-OH ligand. Peaks with an asterisk correspond 1065 to PrKAI2d3 covalently bound to $\mathrm{D}-\mathrm{OH}$. The mass increments were measured for the 1066 PrKAI2d3-D-OH complex, 114.2 Da. Ligand-modified amino acids were identified by 1067 nanoLC-MSMS analyses after Glu-C proteolysis. (j) Fragmentation spectra of unmodified 1068 and different ligand-modified peptides. Labeled peaks correspond to the $\mathrm{b}$ and $\mathrm{y}$ fragments of 1069 the double-charged precursor ion displayed at the top. The D-OH ligand-modified histidine 1070 residue is underlined. 
1072 Supplementary Figure 1. Chemical structures. (a) Natural strigolactones. (b) Profluorescent 1073 probes. GC series and Yoshimulactone (YLG).

1074 Supplementary Figure 2. Phylogenetic analysis of KAI2 and D14 nucleotide sequences. 1075 The phylogenetic tree was constructed with the maximum likelihood method and 1,000 1076 bootstraps replicates by means of RAxML. Scale bar $=0.1$ substitutions per site.

1077 Supplementary Figure 3. Sequence alignment of Phelipanche ramosa (Pr) and $\boldsymbol{P}$. 1078 aegyptiaca (Pa) KAI2 protein with D14 and KAI2 proteins from Arabidopsis (At), rice 1079 (Os), pea (RMS), and the bacterial RbsQ. The three amino acid residues corresponding to 1080 the catalytic triad are marked with asterisks. Amino residues highlighted in the Figure $1 \mathbf{b}$ are 1081 indicated with a blue arrowhead. Amino acid numbers are indicated for AtD14. Note that the 1082 rice OsD14 protein has a non-conserved 50-amino-acid N-terminal extension omitted in the 1083 alignment.

1084 Supplementary Figure 4. Dose response germination stimulant activities of (+)-GR24 and 1085 (-)-GR24 with Arabidopsis lines. Dose response germination stimulation activities and 1086 modeled curves of (+)-GR24 (a) and (-)-GR24 (b) on seeds of Arabidopsis Col-0 and $h t l-3 / \mathrm{p} 35 \mathrm{~S}:: \operatorname{PrKAI} 2 \mathrm{~d} 3$. (c) Half maximal effective concentration $\left(\mathrm{EC}_{50}\right)$. (d) Maximum

1088 germination stimulation activities. Data are indicated \pm SE. (e) Relative accumulation of 1089 PrKAI2d3 transcripts in Arabidopsis Col-0, htl-3, and p35S::GFP-PrKAI2d3 \#4 (htl-3) seeds 1090 imbibed for $24 \mathrm{~h}$. (f) Accumulation of PrKAI2d3 proteins in Arabidopsis p35S::GFP1091 PrKAI2d3 \#4 (htl-3) and htl-3 5d-old seedlings.

1092 Supplementary Figure 5. Germination assay on $\boldsymbol{P}$. ramosa parasitic-plant seeds of GR24 1093 isomers [(+)-GR24, (-)-GR24, (+)-2'-epi-GR24, and (-)-2'-epi-GR24] and methyl 1094 variation on the GR24 D-ring [( \pm )-Desmethyl-GR24, $( \pm)-4^{\prime}-$ Desmethyl-2'-epi-GR24, and 1095 ( \pm )-3'-Me-GR24]. (a) and (c) Dose response germination stimulation activities and modeled 1096 curves.(b) and (d) Maximum germination stimulation activity relative to ( \pm )-GR24 (1 $\mu \mathrm{M})$. 1097 Data are indicated \pm SE.

1098 Supplementary Figure 6. Germination assay on $\boldsymbol{P}$. ramosa parasitic-plant seeds of various 1099 profluorescent ligands and biochemical analysis of the interaction between PrKAI2d3 
1100 and various ligands by DSF. (a) Dose response germination stimulation activities and modeled curves. (b) Maximum germination stimulant activity relative to $( \pm)$-GR24 $(1 \mu \mathrm{M})$.

1102 (c) Half maximal effective concentration $\left(\mathrm{EC}_{50}\right)\left(\mathrm{mol} \mathrm{L}^{-1}\right)$. Data are indicated $\pm \mathrm{SE}$. (d-i) 1103 Melting temperature curves of PrKAI2d3 with $( \pm)-G R 24$ (d), $( \pm)$-GC486 (e), $( \pm)$-GC240 (f), 1104 DiFMU (g), (+)-GC242 (h), and (-)-GC242 (i) at varying concentrations assessed by DSF. 1105 Each line represents the average protein melt curve for three technical replicates and the 1106 experiment was carried out twice.

1107 Supplementary Figure 7. Biochemical analysis of the interaction between PrKAI2d3 (a-d, 1108 i-k) or PrKAI2d3 ${ }^{\text {S98A }}$ (e-h, l-n) and various ligands by DSF. Melting temperature curves of 1109 PrKAI2d3 or PrKAI2d3 ${ }^{\text {S98A }}$ at $10 \mu \mathrm{M}$ with (+)-GR24 (a, e), (-)-GR24 (b, f), (+)-2'-epi-GR24

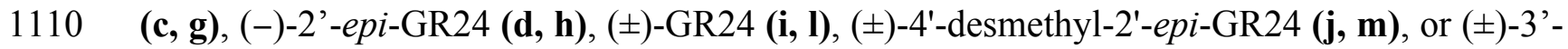
1111 Me-GR24 $(\mathbf{k}, \mathbf{n})$ at varying concentrations assessed by DSF. Each line represents the average 1112 protein melt curve for three technical replicates and the experiment was carried out twice.

1113 Supplementary Figure 8. Intrinsic tryptophan fluorescence of PrKAI2d3 in the presence 1114 of SL analogs. Changes in intrinsic fluorescence emission spectra of PrKAI2d3 in the 1115 presence of various concentrations of (+)-GR24 (a), (-)-GR24 (b), (+)-2'-epi-GR24 (c), (-)2'-epi-GR24 (d), ( \pm )-GR24

(e), ( \pm )-4'-desmethyl-2'-epi-GR24 (f), or ( \pm )-3'-Me-GR24 (g).

1117 Proteins $(10 \mu \mathrm{M})$ were incubated with increasing amounts of ligand $(0-800 \mu \mathrm{M}$ from top to 1118 bottom). The observed relative changes in intrinsic fluorescence were plotted as a function of 1119 the SL analog concentration, transformed to a saturation degree, and used to determine the 1120 apparent $K_{\mathrm{D}}$ values relevant to Figure $2 \mathbf{h}, \mathbf{i}$. The plots represent the mean of two replicates and 1121 the experiments were repeated at least three times. The analysis was done with GraphPad 1122 Prism 8.0 Software.

1123 Supplementary Figure 9. Intrinsic tryptophan fluorescence of PrKAI2d3 in the presence

1124 of profluorescent SL probes. Changes in intrinsic fluorescence emission spectra of $1125 \operatorname{PrKAI} 2 \mathrm{~d} 3$ in the presence of various concentrations of $( \pm)-G C 486(\mathbf{a}),( \pm)-G C 240(\mathbf{b})$, 1126 DiFMU (c), YLG (d), ( \pm )-GC242 (i), (+)-GC242 (j), or (-)-GC242 (k). Proteins $(10 \mu \mathrm{M})$ 1127 were incubated with increasing amounts of ligand $(0-800 \mu \mathrm{M}$ from top to bottom). The 1128 observed relative changes in intrinsic fluorescence were plotted as a function of the SL analog 1129 concentration and transformed to saturation. Plots of fluorescence intensity versus $( \pm)$-GC486 1130 (e), $( \pm)$-GC240 (f), DiFMU (g), and YLG concentrations used to determine the apparent $K_{\mathrm{D}}$ 
1131 values. The plots represent the mean of two replicates and the experiments were repeated at

1132 least three times. The analysis was done with GraphPad Prism 8.0 Software.

1133 Supplementary Figure 10. PrKAI2d3 hydrolysis activity. Progress curves during the 41134 nitrophenyl acetate $\left(p\right.$-NPA) $(1 \mathrm{mM})$ hydrolysis by PrKAI2d3, PrKAI2d3 ${ }^{\mathrm{S} 98 \mathrm{~A}}$, AtKAI2, and 1135 AtD14 $(4 \mu \mathrm{M})$. The $p$-NPA release was monitored $\left(\mathrm{A}_{405}\right)$ at $25{ }^{\circ} \mathrm{C}$.

1136 Supplementary Figure 11. Biochemical analysis of the interaction between PrKAI2d3 and

1137 ITCs by DSF. Melting temperature curves of PrKAI2d3 at $10 \mu \mathrm{M}$ with ( \pm )-GR24 (a), 21138 PEITC (b), or BITC (c) at varying concentrations assessed by DSF. Each line represents the 1139 average protein melt curve for three technical replicates and the experiment was carried out 1140 twice.

1141 Supplementary Figure 12. Germination inhibition by various chemicals and stimulation 1142 by D-analogs. Dose response germination stimulation (GS) activities with $10 \mathrm{nM}$ GR24 (a) 1143 and $100 \mathrm{nM}$ 2-PEITC (b) and modeled curves (c) and (d). Maximum of germination 1144 stimulation activity relative to $( \pm)-G R 24(1 \mu \mathrm{M})$. Data are indicated \pm SE.

1145 Supplementary Figure 13. Bud outgrowth inhibition activity assay for D derivatives after 1146 direct stem infusion. Data are means $\pm \mathrm{SE}(n=12), 8$ days after treatment of the pea plants 1147 rms $1-10$ and Térèse as control. $* P<0.5, * * * P<0.001$, Kruskal-Wallis rank sum test, 1148 compared to control values (CTL0).

1149 Supplementary Figure 14. Perception of the germination stimulant D-OH by ShHTL7.

1150 Dose response germination stimulation activities and modeled curves of $( \pm)$-GR24 and D-OH 1151 on seeds of S. hermonthica (a), Arabidopsis Col-0 (c), and Arabidopsis htl-3, ShHTL7 (34 ${ }^{\circ} \mathrm{C}$, 1152 continuous light for Arabidopsis (d). (b) Seed germination of Arabidopsis Col-0, htl-3, htl1153 3/ShHTL7, and $\max 2$ with GR24 and D-OH $(10 \mu \mathrm{M})$. (e) Half maximal effective 1154 concentration $\left(\mathrm{EC}_{50}\right)$. (f) Maximum germination stimulation activities. Data are indicated \pm 1155 SE.

1156 Supplementary Table $1 . \mathrm{EC}_{50}$ and maximum germination percentage of (+)-GR24, and (-)1157 GR24 and of $( \pm)-G R 24$ and D-OH in Arabidopsis lines.

1158 Supplementary Table $2 . \mathrm{EC}_{50}$ and maximum of germination stimulant activity of compounds 1159 for P. ramosa. 
bioRxiv preprint doi: https://doi.org/10.1101/2020.06.09.136473; this version posted June 10, 2020. The copyright holder for this preprint (which was not certified by peer review) is the author/funder. All rights reserved. No reuse allowed without permission.

1160 Supplementary Table $3 . \mathrm{IC}_{50}$ and maximum of inhibition of putative inhibitors of PrKAI2d3

1161 for P. ramosa.

1162 Supplementary Table 4. Oligonucleotides used. 


\section{A Phelipanche ramosa KAI2 Protein Perceives enzymatically Strigolactones and Isothiocyanates}

Alexandre de Saint Germain, ${ }^{a}$ Anse Jacobs, ${ }^{b-e}$ Guillaume Brun, ${ }^{f, g}$ Jean-Bernard Pouvreau, ${ }^{f}$ Lukas

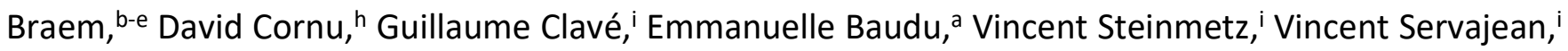
Susann Wicke, ${ }^{g}$ Kris Gevaert, ${ }^{d, e}$ Philippe Simier, ${ }^{f}$ Sofie Goormachtig, ${ }^{b, c}$ Philippe Delavault ${ }^{f}$ and FrançoisDidier Boyer ${ }^{*}$ 
bioRxiv preprint doi: https://doi.org/10.1101/2020.06.09.136473; this version posted June 10, 2020. The copyright holder for this preprint (which was not certified by peer review) is the author/funder. All rights reserved. No reuse allowed without permission.

a

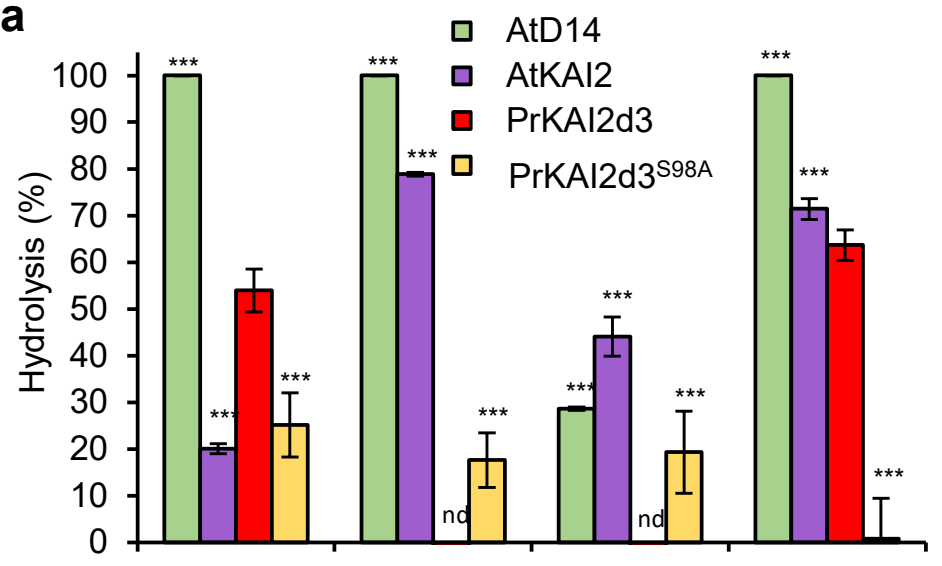

$(+)$-GR24 (-)-GR24

$(+)-2-e p i-G R 24 \quad(-)-2 ;-e p i-G R 24$
C

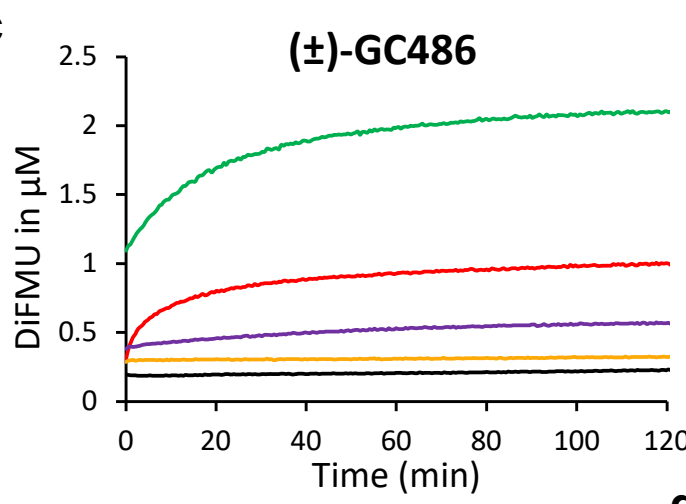

g

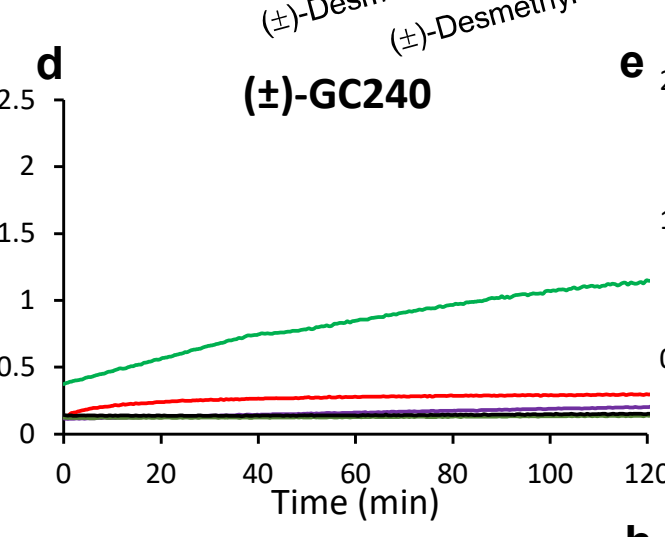

i
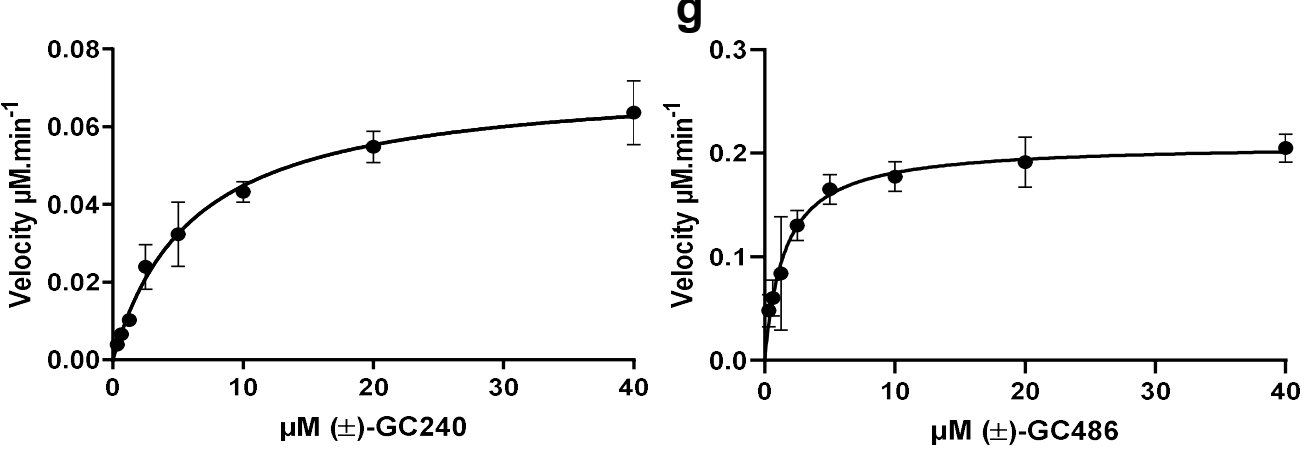

h b

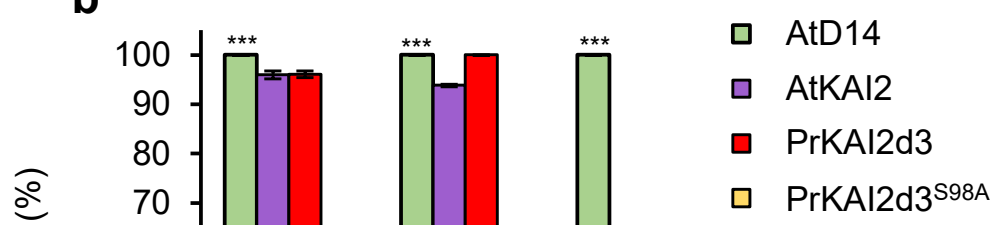

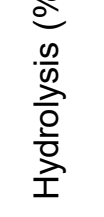

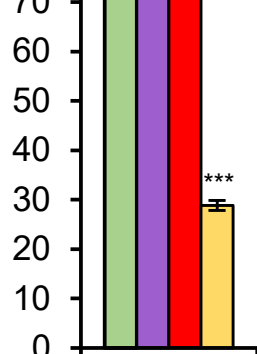

-GR24
$( \pm)-G R 24$

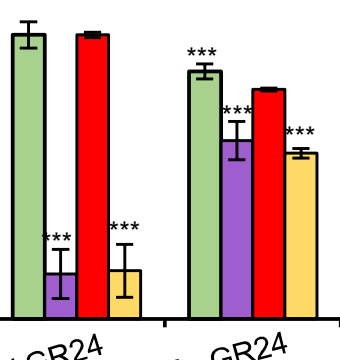

$\pm)^{2}-2^{2}-\mathrm{P}^{\mathrm{p}-G R 2^{4}}( \pm)^{-3}-M e^{-G R 24}$
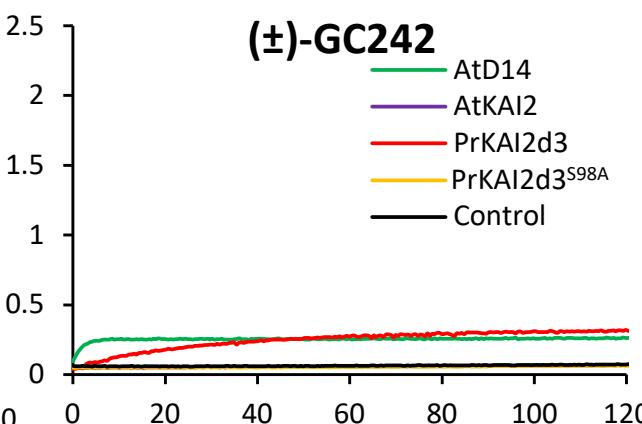

Time (min)

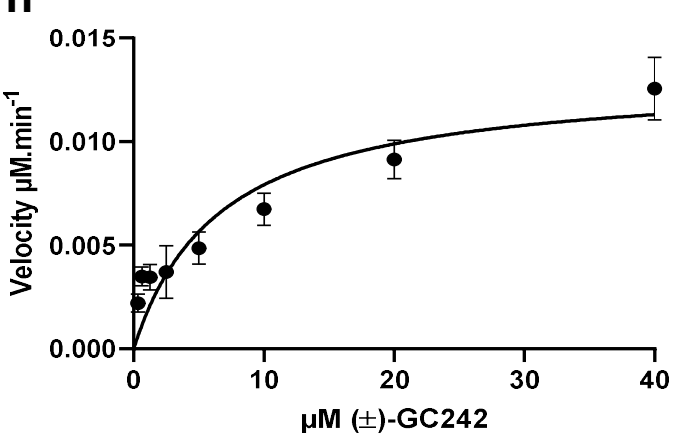
Protein - molecule
PrKAI2d3 - ( \pm )-GC486
PrKAI2d3 - ( \pm )-GC240
PrKAI2d3 - ( \pm )-GC242
$K_{1 / 2}(\mu \mathrm{M})$
$1.536 \pm 0.283$
$6.139 \pm 0.866$
$6.665 \pm 1.883$
$V_{\text {max }}\left(\mu \mathrm{M} \min ^{-1}\right)$
$0.2090 \pm 0.0094$
$0.07231 \pm 0.00345$
$0.01318 \pm 0.00129$
$k_{c a t}\left(\min ^{-1}\right)$
$0.6332 \pm 0.0286$
$0.2191 \pm 0.0104$
$0.03995 \pm 0.00390$

j
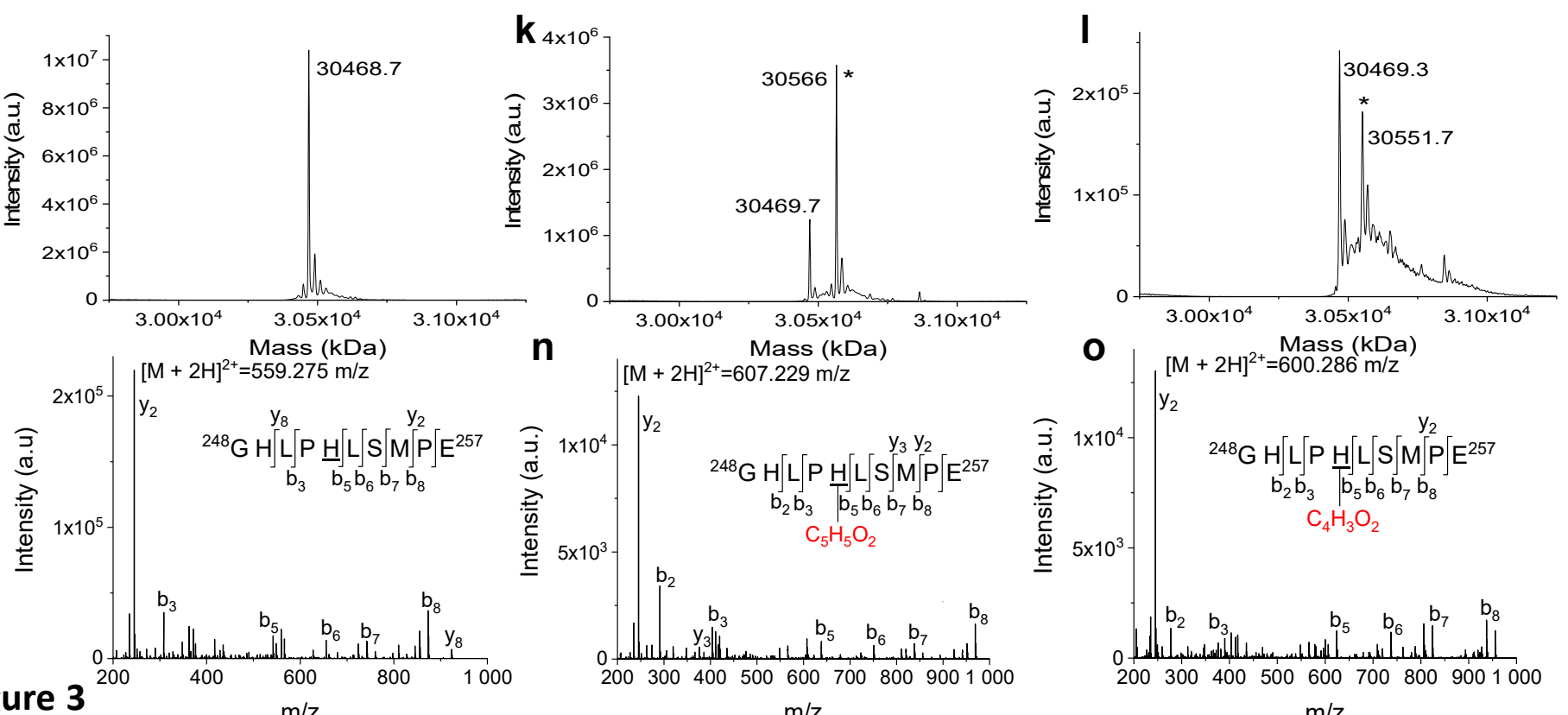

$$
\text { 京 }
$$

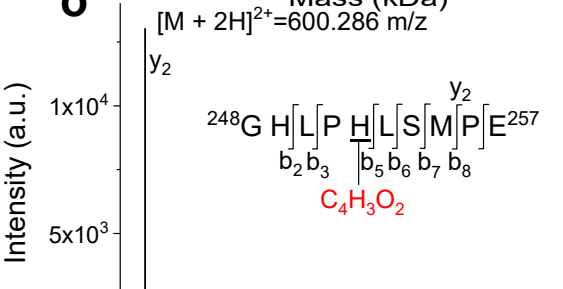


a<smiles>S=C=NCCc1ccccc1</smiles>

2-PEITC<smiles>S=C=NCc1ccccc1</smiles>

b

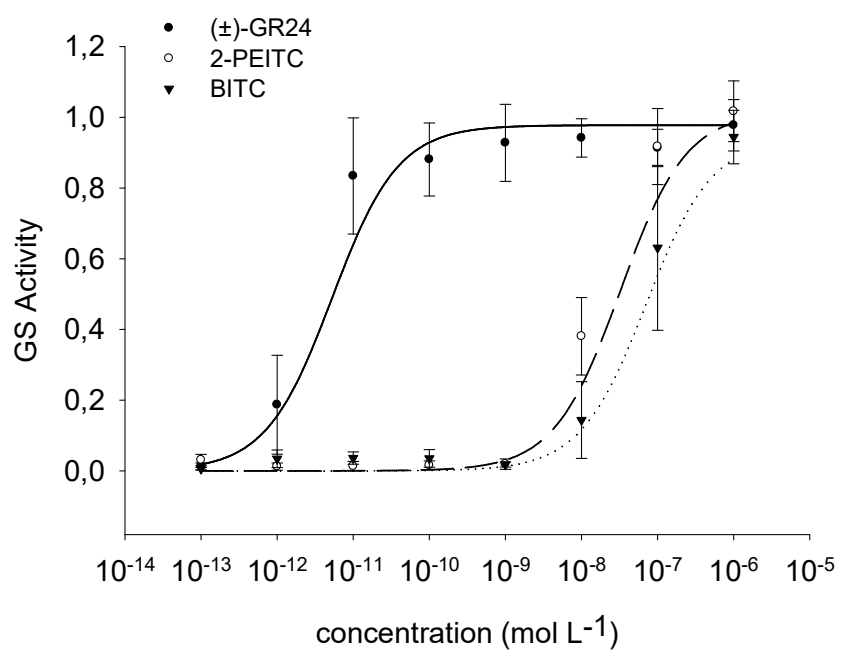

C

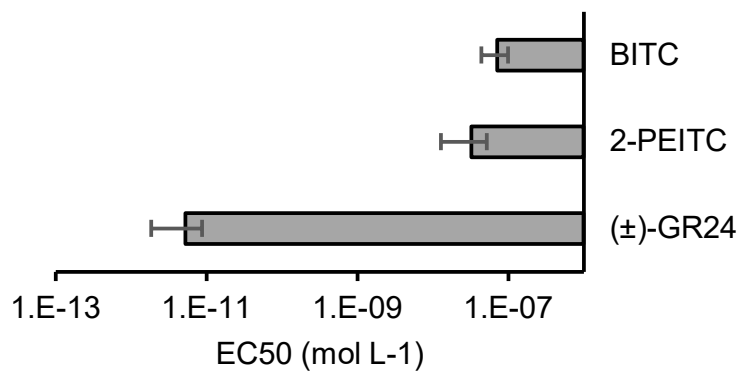

g
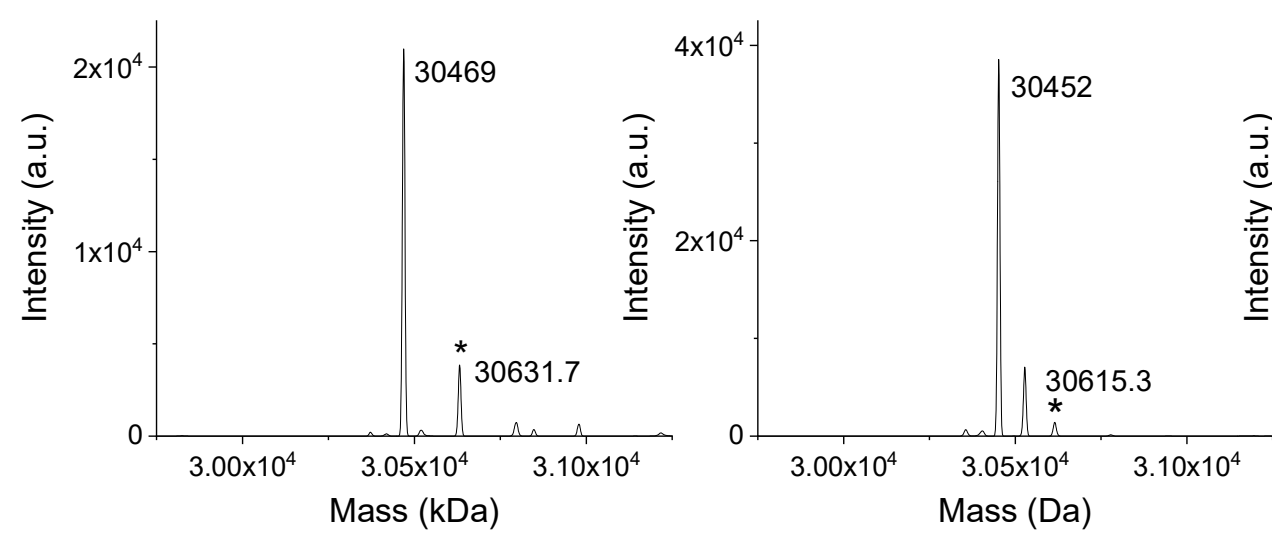
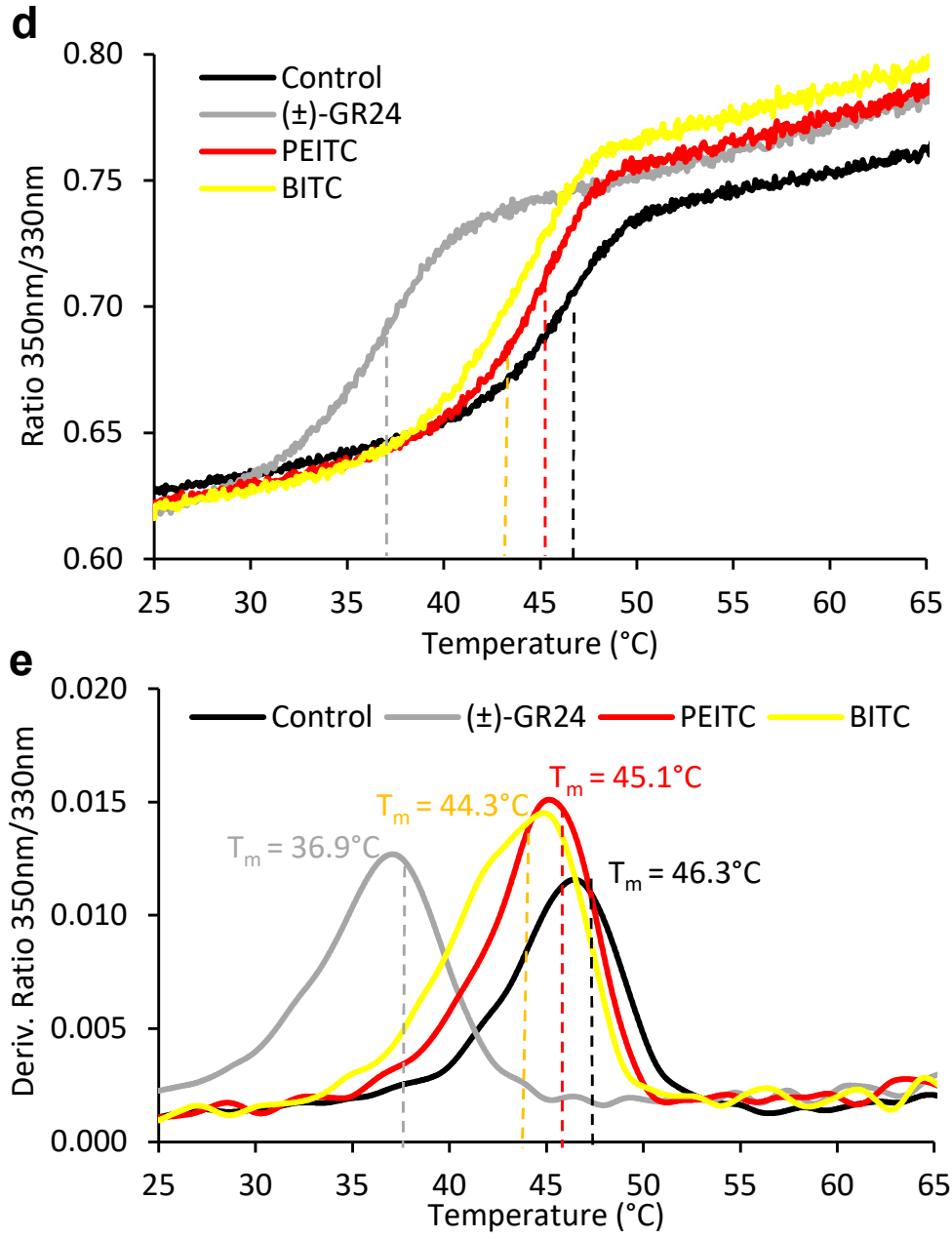

$\mathbf{f}$

i
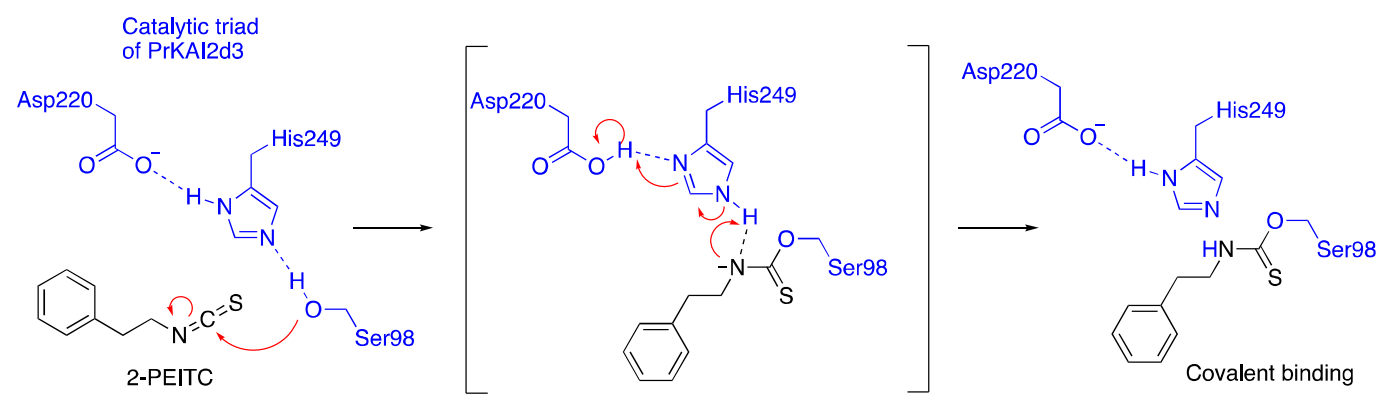

\section{h}

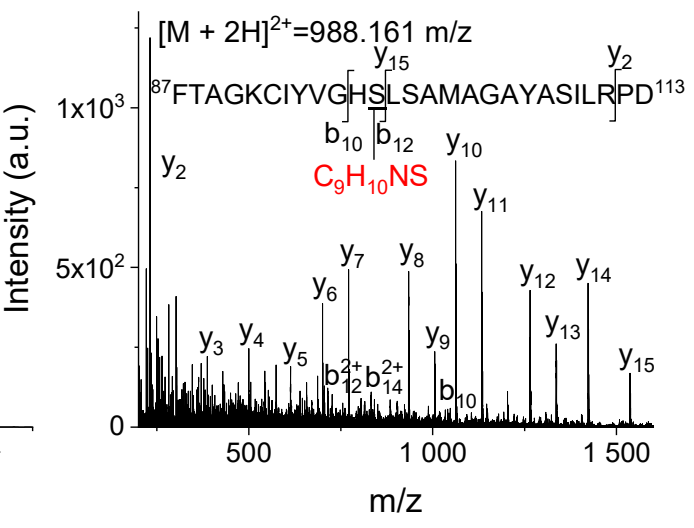


